\section{Cómo se construye una torre mudéjar. La torre de la iglesia de San Pedro el Viejo de Madrid}

\author{
Luis Caballero Zoreda*, José Ignacio Murillo Fragero \\ * $\mathrm{IH}, \mathrm{CSIC}$
}

\begin{abstract}
Resumen
La torre mudéjar de S. Pedro el Viejo de Madrid ofrece, gracias a diversas intervenciones de época moderna y contemporánea, la posibilidad de analizar su desconocido proceso de construcción y sus técnicas constructivas donde se combinan fábricas de ladrillo y calicanto. Un muro de ladrillo con barro sirvió de encofrado de la fábrica de calicanto y luego fue utilizado como base de los primeros tramos de la escalera. También se utilizaron como encofrado la madera y los muros de ladrillo visto.
\end{abstract}

Palabras clave: Técnicas constructivas. Mudéjar toledano. Torre, escalera, encofrado, ladrillo, calicanto. Madrid, S. Pedro el Viejo.

\begin{abstract}
The Mudejar tower of San Pedro el Viejo de Madrid offers, thanks to various modern-era and contemporary interventions, the possibility of analyzing its unknown construction process and construction tecniques combining brick and limestone stonework. A wall of brick and mud was used as the formwork of limestone walls and then used as a base for the first sections and staircase. The wood and brick walls on view were also used as formwork.
\end{abstract}

Key words: Construction techniques. Toledo Mudejar. Tower, staircase, formwork, brick, limestone. Madrid, San Pedro el Viejo.

\section{PLANTEAMIENTO. LA DIVERGENCIA ENTRE LA OBSERVACIÓN DE LOS DATOS Y LA ACEPTACIÓN DE SU EXPLICACIÓN}

Una de las virtudes de la aplicación del Sistema de Registro Estratigráfico (Método Harris) al estudio de los edificios históricos, considerados por tanto como objetos de estudio de la arqueología, es el descubrimiento de modos constructivos hasta el momento desconocidos y que, de no emplear este modelo de análisis, pueden pasar desapercibidos. La aplicación de este sistema de trabajo obliga a la inspección personal y sistemática del edificio, evidenciando todos sus elementos y sus relaciones contextuales (o, lo que es lo mismo, los elementos que conforman sus estructuras), otorgando a unos y otras el mismo valor. Incidir en estas cuestiones, de sobra conocidas, puede considerarse inútil pero, en la práctica, redundan en la aceptación de los resultados de la lectura de paramentos.

El empleo habitual de esta metodología permite comprender el rigor que conlleva por la sistematización de la observación y del registro de lo observado. Sin embargo, quien contempla desde fuera los resultados obtenidos con este método, si no es consciente de las características del sistema empleado, puede desestimarlos. Esta situación, según nuestra experiencia, se produce en más ocasiones de lo que sería de desear. Entonces, el espectador externo arrastra con su duda a los datos que deja de considerar evidentes para creerlos fruto de una observación subjetiva. Tiende a confundir dos niveles perfectamente diferenciados: el del registro, obtenido con un sistema más riguroso y sistemático que con otros métodos hasta ahora vigentes y aún en uso, y el de la explicación, un nivel que ya no depende del rigor, sino de la objetividad (o subjetividad) de la explicación, ya sea una explicación histórica o constructiva (esto es, ya competa a la Historia o a la Arquitectura). Si su observación (menos rigurosa y sistemática) le lleva a una conclusión diferente, puede que siga apegado al nivel de los modelos, rechazando los conseguidos, dudando de la toma de datos y ampliando su duda hasta llegar a discutir el método por considerarlo confuso, complejo e inapropiado.

En nuestro caso, somos conscientes de la diferencia entre el nivel de la observación y el registro y el de la explicación. Lo que exponemos a continuación es ejemplo de ello. La observación meticulosa y sistemática ofrece unos datos que conducen a un modelo, en este caso constructivo. Ello no quiere decir que, sin renunciar a lo observado, no seamos conscientes de la «dureza» del modelo obtenido y de los problemas que presenta. Solo la multiplicación de observaciones, conseguidas rigurosamente, contrastables y compatibles entre sí por haberse obtenido con el mismo método, podrá ir solucionando las aparentes contradiccio- 
nes de los modelos explicativos, sin tener por ello que corregir las observaciones realizadas.

Sin embargo, se puede incurrir en errores a la hora de observar y recoger los datos. Por ello nuestra obligación es ofrecer el registro al contraste científico de tal modo que se pueda rehacer el proceso de observación, de acuerdo con una norma común y consensuada (que es lo que convierte nuestro trabajo en una profesión) que permita descubrir dónde erramos. La descripción con sus fichas o listados; el sistema contextual de relaciones con sus diagramas; y la ubicación de los elementos (si no también las relaciones, como propone Doglioni) con la planimetría; y, hasta donde sea posible, con el mayor detalle (a nivel de Unidades Estratigráficos mejor que al de Actividades) e indicando dónde se han archivado los documentos con el fin de poder consultar los originales.

\section{DATOS HISTORIOGRÁFICOS Y FINALIDAD DEL ESTUDIO}

La iglesia de $S$. Pedro pasa por ser una de las más antiguas de la ciudad de Madrid (fig. 1). Su existencia se remontaría a fines del s. XII (carta de 1194 y Fuero de Madrid probablemente de 1202; Fita 1886: 78, doc. n. $\left.{ }^{\circ} 15\right)$. Incluso se pretende que fuera anterior a la reconquista del lugar y aún se mantiene que su torre fue el alminar de una mezquita $^{1}$, lo que ha hecho que se propusieran distintas fases cronológico-culturales en ella (Íñiguez 1971). También se asegura que la iglesia original estaba ubicada más arriba de donde está la actual, lo que quizás se explique por el intento de hacerse con los derechos de las aguas de las fuentes de San Pedro o de los Caños Viejos en el s. XVI (Quintana 1629: 70); lo que, por otra parte, ha dado lugar a que otro autor pusiera en relación su construcción con la fundación legendaria de su feligresía por Alfonso XI (1344; Tormo 1927: 46-47).

No son muchos los estudios que se han dedicado a este edificio (Gómez Moreno 1927; Tormo 1927; Torres Balbás 1949; Aurea 1983; Pavón, 1984/85; Montero 1987 y 1990; Nuere 1994), siendo Concepción Abad (1991, vol. 2, 134-140) quien mayor atención le ha prestado. Esta autora, analizando el conjunto del templo, muy transformado por remodelaciones y ampliaciones a lo largo del tiempo, consigue reconocer las partes más antiguas en diferentes zonas: el tramo recto que precedía el ábside, los pilares de separación de las naves, partes importantes de los muros norte y sur y la torre. Esta última se divide en tres niveles, todos coetáneos, incluido el cuerpo de campanas

\footnotetext{
1 Resumen de la discusión en Abad, 1991: 1, 177-178, rechazando esta adscripción para la de $S$. Pedro, pero manteniendo la duda para la constructivamente gemela de $\mathrm{S}$. Nicolás.
}

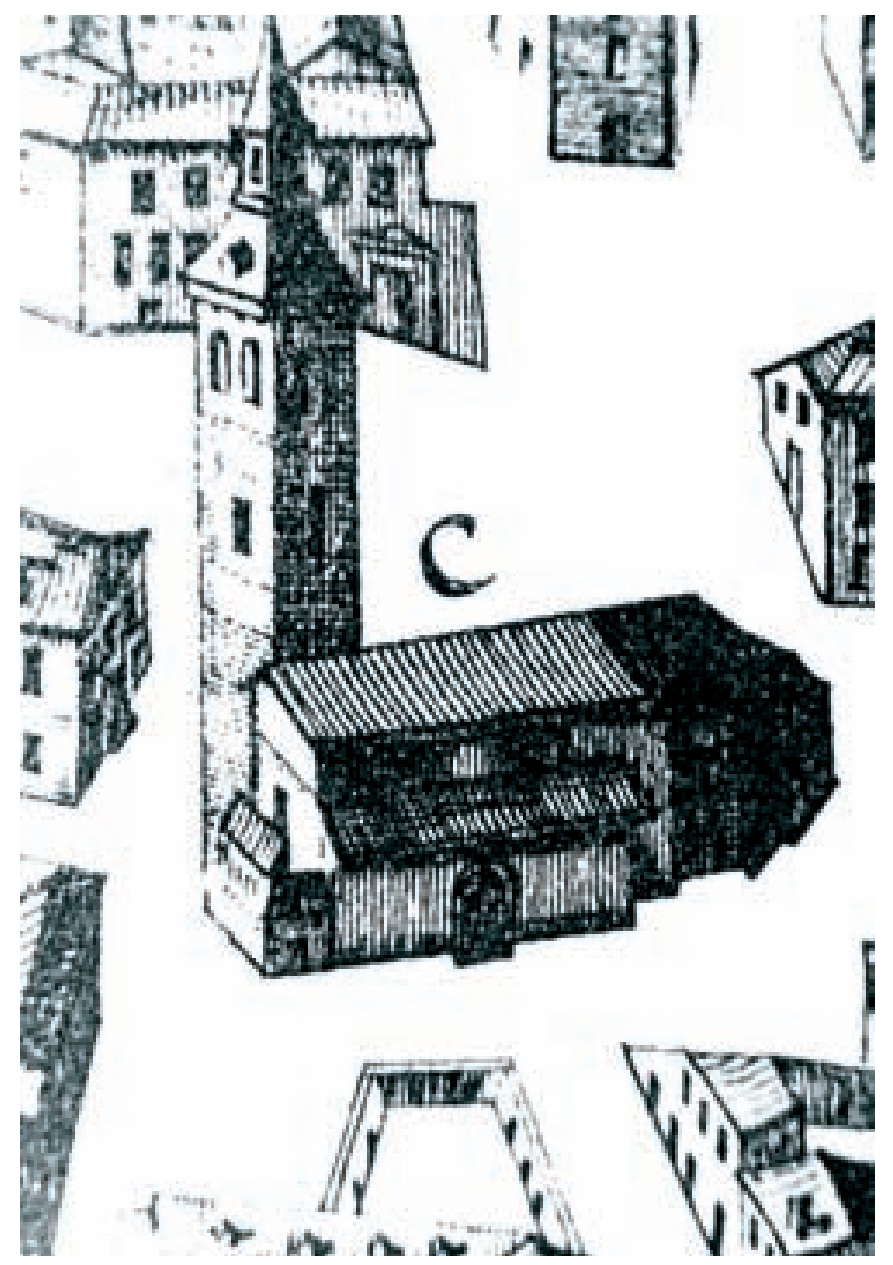

Fig. 1. S. Pedro el Viejo de Madrid según el plano de Madrid de Teixeira, 1656

en el que, no obstante, piensa hubo una mala restauración que alteraría la fisonomía de los arcos, dando la sensación de etapas diferentes. Abad considera en su trabajo que todo lo conservado de la antigua iglesia y la torre responde a un mismo esfuerzo constructivo que, por sus paralelos arquitectónicos y decorativos, se fecharía a comienzos del siglo XIII (vol. 1, 177-178).

Los estudios del arquitecto José Miguel Ávila son los que más interés tienen para nosotros por describir su estado, plantear algunas preguntas sobre él e informar del objetivo de las obras. Su relación con el edificio se inició en 1991, cuando, ante las grietas abiertas en la fábrica de la torre y tras un Decreto de la Gerencia Municipal de Madrid requiriendo al Arzobispado la consolidación y estudio de sus movimientos, la Comunidad de Madrid le encarga un Informe (1993). En él justifica que el edificio está estabilizado, por lo que en 1999 propone un proyecto de investigación que estudie las razones de las grietas. Además de la descripción estructural, nos da noticias sobre las intervenciones efectuadas, como el metro y medio que se rebajó la cota de la calle Costanilla 
de San Pedro, que corre por su costado Oeste, a mediados del s. XIX; pequeñas obras efectuadas tras la guerra civil; la restauración de Amparo Berlinches efectuada hace más de diez años (hacia 1987 por tanto; en la que, como veremos, se debe inscribir el intento por recuperar la escalera desaparecida en la planta inferior); y la colocación de testigos para control de los agrietamientos el 19 de abril de 1990. La torre está girada y desplomada entre 50 y $70 \mathrm{~cm}$ hacia Oeste y posee grietas observables en sus caras exteriores Sur y Norte. Estas grietas considera que están estabilizadas como mínimo desde las obras de hace 150 años; no son sólo consecuencia de este giro, ni son recientes, sino que ocurrieron hace siglos. Estudia posibles causas por las que pudieron producirse: la descompresión al vaciar la calle; la presión producida por la tierra que rellena la parte baja del interior, quizás aumentada al inundarse de agua; los efectos producidos por la caída de un rayo; y el balanceo de la gran campana central. Prácticamente todas las desecha, excepto la del terreno sobre el que se asienta (para el giro) y la tierra interior y la de la campana para las grietas. Para las dos mayores, que llegan a la altura de la cumbre de la iglesia, también considera la posible existencia de huecos bajo rasante como criptas o socavones.

Otras observaciones se pueden considerar como preguntas para nuestro análisis (Âvila 1998: 15):

— si el acceso actual es nuevo o ha sido ensanchado, pues posee un cargadero de madera;

- si la escalera ha sido desmantelada en la parte inferior, hasta el acceso al coro al que se sube por un talud en el terreno (en realidad, como veremos, es un muro de ladrillo y barro, desconocido entonces por la abundante suciedad que lo cubría);

—si se diferencia, en el interior, la parte inferior, tosca, sin desbastar y como revestida de un mortero de cal, de la superior, con paramento de ladrillo correctamente aparejado.

— si en la parte inferior hubo o no hubo escalera o si ésta pudo bajar aun más hasta posibles sótanos o criptas cegadas.

Su proyecto propone, entre otros objetivos, éste referente a nuestro estudio: «Esta labor historiográfica se completa arqueológicamente con el estudio de los elementos del subsuelo, con la lectura e interpretación de paramentos y con un estudio de los dos materiales que conforman la torre: ladrillos y madera. Su estudio busca un seguimiento de las etapas constructivas de la torre a través de sus materiales y paramentos interiores y exteriores» (Ávila 1998: 19).

La lectura de paramentos realizada se ha visto también en cierta manera motivada por nuevas explicaciones del arquitecto, expuestas en conversaciones con nosotros y conocidas y apoyadas parcialmente por la historiadora Abad. En su opinión pueden preexistir restos de una torre anterior de ladrillo de época musulmana (nuestro «muro de ladrillo y barro»), acorde con cierta tradición histórica del edificio. En esta torre se apoyaría otra de calicanto (los muros perimetrales de las plantas $1 .^{\mathrm{a}}$ y $2 .^{\mathrm{a}}$ ) de época mudéjar, también anterior a la iglesia dada su posición descentrada y algo girada. Finalmente, otra tercera obra de ladrillo visto (las fachadas) forraría su alzado exterior dándole su aspecto actual. Esta secuencia se basaría en la diferencia reconocible en los materiales constructivos y su aparejo.

El encargo de nuestro estudio tuvo lugar en el año 2002 por el arquitecto José Miguel Ávila. Se centró en la lectura de paramentos de la torre y su relación con la iglesia, de acuerdo con la petición del arquitecto, el «seguimiento de las etapas constructivas» (Ávila 1998: 19). Sin embargo, en este trabajo nos vamos a centrar especialmente en los problemas surgidos al explicar cómo se construyó, refiriéndonos solo de modo breve a su secuencia histórica.

Además de la lectura de paramentos se han efectuado análisis de tipología de materiales y aparejos y se han tomado muestras de argamasas, concordes con el estudio estratigráfico, para el análisis comparativo de su composición. Somos conscientes de que estos trabajos son incompletos, debiendo ampliarse con un análisis mucho más complejo formado cuando menos con la lectura y la excavación arqueológica del edificio de la iglesia, con el estudio de los documentos históricos y con los análisis de los ladrillos y las maderas. Pero lo que se convino era lo que se ajustaba a las posibilidades del momento dentro del proyecto de restauración de la torre. En cualquier caso, hemos de reconocer y agradecer el correcto planteamiento del arquitecto restaurador y la comprensión y ayuda que siempre nos prestó dentro de las severas limitaciones a que se vio sometido el trabajo $^{2}$.

\footnotetext{
${ }^{2}$ Los trabajos se efectuaron bajo un convenio de colaboración entre el Instituto de Historia del Consejo Superior de Investigaciones Científicas y la dirección facultativa del arquitecto José Miguel Âvila Jalvo, debido a su iniciativa, y financiado por el Arzobispado de Madrid. Los trabajos fueron dirigidos por Luis Caballero Zoreda y realizados por Fernando Arce Sainz y M. ${ }^{a}$ de los Ángeles Utrero Agudo, con la colaboración de José Ignacio Murillo Fragero y Mario Núnez Herrero. El análisis de los morteros fue efectuado por Blanca Guarás (2002). Se utilizó la planimetría puesta a nuestra disposición por el arquitecto. El estudio de las maderas será efectuado por Eduardo Rodríguez Trobajo del Laboratorio de Dendrodatación del INIA y Antonio Rubinos del Laboratorio de Radiocarbono del Instituto Rocasolano del CSIC, Madrid. La documentación original se encuentra depositada en el IH del CSIC, Madrid. Agradecemos a Miguel Ángel Tabales sus indicaciones. Nuestros planos y perspectivas dependen de los planos puestos a nuestra disposición por el arquitecto.
} 


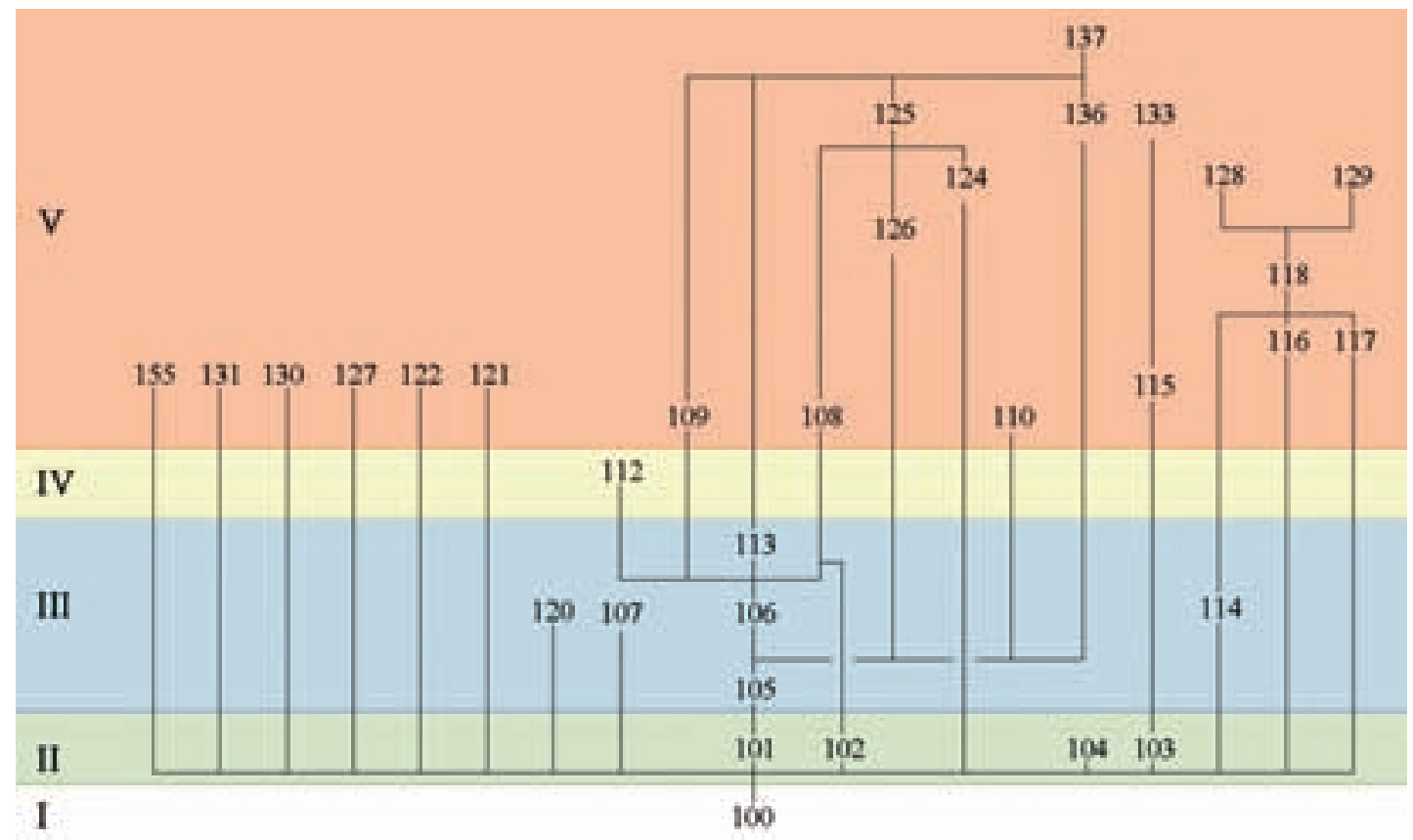

Fig. 2. Diagrama de actividades. S. Pedro el Viejo de Madrid

\section{SECUENCIA HISTÓRICO-CONSTRUCTIVA}

La torre, de treinta metros de altura, está formada por un machón central de planta cuadrada rodeado por un muro exterior de planta también cuadrada de cinco metros de lado (Ávila 1998: 14). La escalera corre entre ambos, apoyada en plataformas horizontales de madera, que denominamos impropiamente mesetas, descargadas en el machón y el muro perimetral ${ }^{3}$. Se sitúa en el extremo occidental de la iglesia, sobresaliendo medio cuerpo del testero; incluida en la nave central pero descentrada a su lado norte de modo que su cara de ese lado se alinea con la arquería septentrional de la iglesia (fig. 16 y 17).

La síntesis de la documentación obtenida durante el análisis del edificio nos ofrece una secuencia históricoconstructiva (fig. 2) donde la torre forma parte de la etapa I, como una pieza más del proyecto edilicio del templo mudéjar al que pertenece. Torre e iglesia se construyeron a la vez, como veremos, de modo que el último arco de la arquería norte de la iglesia enjarja con la obra de la torre. A su vez, toda la obra de la torre forma un cuerpo unitario [A100] desde el nivel de cimientos hasta el cierre del campanario, con sus arcos y cornisa incluidos. Ambos hechos se demuestran por la unidad de la fábrica de ladrillo y de calicanto, deducida de la lectura arqueológica; la unidad de la argamasa utilizada; la disposición regular del

\footnotetext{
${ }^{3}$ El único paralelo conocido de escalera sobre techo plano es el de la torre también madrileña de S. Nicolás, Abad 1991: 2, 132, de cronología discutida entre islámica y del s. XII avanzado, fecha esta última en la que parece datarse el templo.
}

aparejo; y la dimensión constante de los ladrillos y de sus lechos y juntas.

Las transformaciones posteriores se limitan a reparaciones puntuales o a la apertura de vanos que no afectan a su morfología, por lo que la torre actual corresponde en gran medida a la de época original. Las dos siguientes etapas son posteriores al impulso constructivo original y constituyen adaptaciones a los nuevos usos que requería la vida del templo. Las dos últimas hacen referencia a lo sucedido durante el siglo XX.

La datación de las etapas II y III es relativa y, por lo tanto, la adscripción de las distintas unidades a cada actividad es meramente estratigráfica, ya que por tipología (cuestiones estéticas, formales o tecnológicas) o por fuentes documentales (extraídas de la bibliografía), no se pueden fijar las actividades a un horizonte cronológico absoluto.

En la etapa II (situada en un amplio abanico cronológico, entre los siglos XIII al XV), tan solo destacamos dos restauraciones, que no representan ninguna importante variación del programa original. Por un lado tenemos la construcción de un forro de ladrillo en la jamba oeste de la puerta de acceso a la torre [A101] que corta y se adosa a la esquina sureste del machón que aquí, en el arranque de la torre, se prolonga hasta el muro sur donde se abre dicha puerta (fig. 7). Creemos más plausible que esta obra pertenezca a una reforma de la obra original, reforzando el marco de la puerta donde las presiones del machón y del muro de fachada eran más importantes.

La segunda restauración corresponde a la colocación de cuatro vigas soleras en la base del campanario [A103] que sustituyen a otras anteriores [A100 UE1092]. En su relación 
I?<smiles>O=C=C1C=C=CC1</smiles>
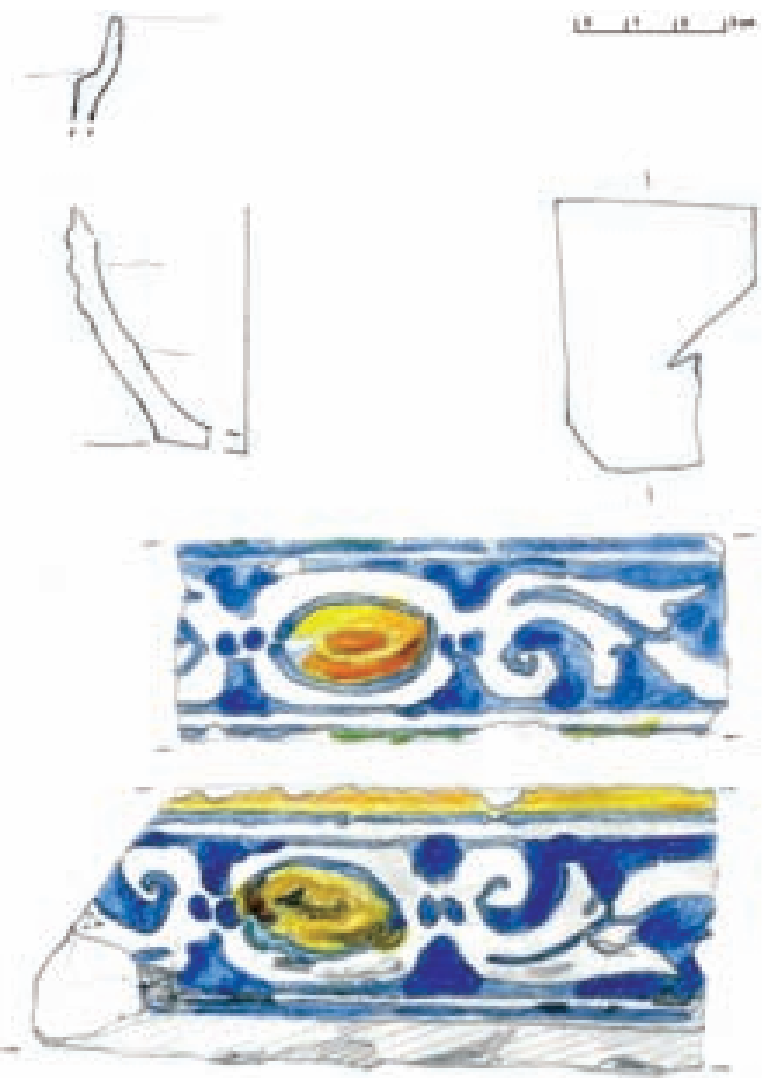

Fig. 3. Cerámicas aparecidas en la restauración de la base del campanario [A103] y azulejo de Talavera aparecida en el relleno del corte del paso al coro moderno [A106]

se encontraron fragmentos cerámicos fechables en el s. XIII o de su tradición (fig. 3. Retuerce 1998, formas E 11 y F).

La etapa III (época moderna) corresponde a una adecuación de la torre como zona de comunicación en el itinerario litúrgico entre la iglesia y el coro alto construido a sus pies. Para ello se remodeló su escalera desde la entrada hasta la nueva puerta del coro alto, ensanchando su hueco a expensas de cortar la superficie del machón central (fig. 4, 5, 7 y 8) y rebajando su nivel para lo que se desmontó la escalera original suavizando su trazado, muy vertical en esta parte (fig. 4 y 11 [A105 UE1001, A107]). Además se abrió el nuevo acceso al coro (fig. 4 [A105 UE1034 4] ), se adosó un muro a la cara externa de su muro oriental (fig. 13 a 16), se abrió una ventana en el muro oeste de la primera planta [A168] y se enfoscó el primer tramo de escalera [A106].

\footnotetext{
${ }^{4}$ La cubierta del actual paso al coro buza de manera muy fuerte, en vez de ir horizontal como era perfectamente posible. Por ello suponemos que esconde dos subetapas, una primera (que solo se podría demostrar si se «excava» una de sus jambas) en que bajaría desde el rellano más alto de la escalera original hasta el nivel del nuevo coro; y una segunda que corresponde a la remodelación de la escalera para suavizar su inclinación, creándose un nuevo rellano a nivel del coro.
}

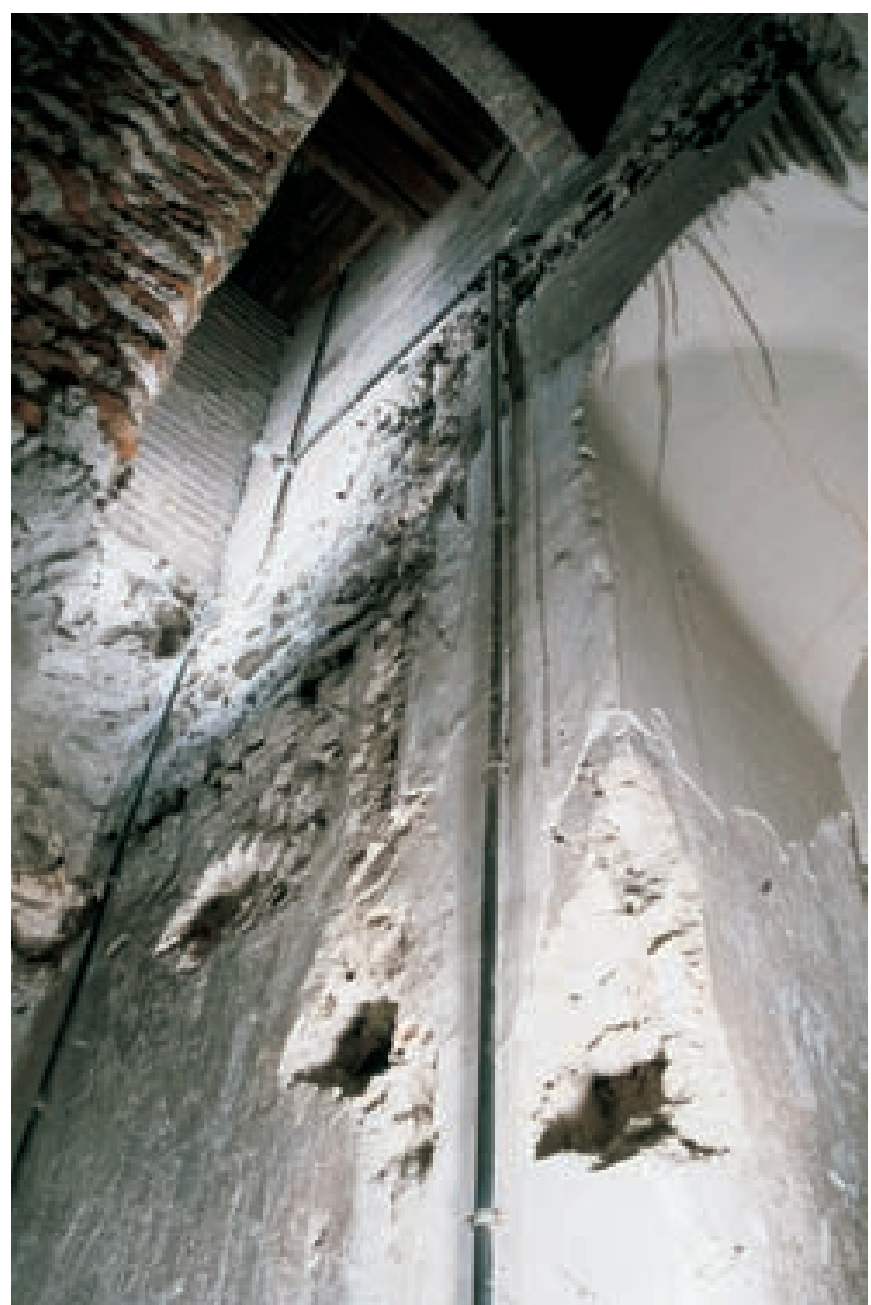

Fig. 4. La entrada al coro moderno [A105 UE1034], planta 2.a, muro E. En primer término el paso al coro con su cubierta buzada. En la pared perimetral los huecos de los atoques originales, indicando el rebaje moderno del nivel de escalera. A la izquierda el muro de ladrillo del machón central con el corte de época moderna. En los muros perimetrales, originales, se puede adivinar el paso de la obra de calicanto a la de ladrillo

Sin atrevernos a ser categóricos, fechamos esta etapa en época moderna. En una pequeña cata abierta en el acceso al coro apareció un azulejo decorativo de Talavera de la Reina (fig. 3) que fecha esta apertura inmediata a la segunda mitad del s. XVI [A106]. Tormo (1927: 48) menciona una reconstrucción del templo en la primera mitad del XVII a cargo del arzobispo de Bríndisi Lorenzo Reinoso, que podría estar en directa relación con lo descrito.

Consideramos como etapa IV lo que fue una acción constructiva coyuntural: las grietas producidas por el movimiento de la torre [A112]. Hay noticia de que en el siglo XIX se rebajó sensiblemente el nivel de la calle Costanilla de San Pedro que pasa por delante de la fachada oeste de la iglesia. Este hecho ha permitido suponer que las grietas fueron la manifestación del desmonte de tierras a los pies 
de la cara oeste de la torre que provocó el desplome del muro perimetral en esa dirección (Ávila 1993 y 1998). Enfrentada esta hipótesis a nuestra lectura estratigráfica podemos decir que tiene visos de credibilidad pues cortan el enfoscado de la etapa III y están cubiertas por las actividades de la etapa V. Se trata de grietas «muertas» ya que el movimiento se produjo de una vez deteniéndose de inmediato al reestablecerse el equilibrio.

La etapa $V$ abarca el último momento de uso del s. XIX $\mathrm{y}$ las restauraciones iniciadas en el s. XX.

Antes de que comenzaran las diferentes obras de «restauración", obligadas por el abandono de la torre al perder las funciones de campanario y subida al coro, nos encontramos con burdas intervenciones que tan sólo buscan solucionar cuestiones funcionales, como la apertura de una puerta [A108] que hoy da al local ocupado por la Cofradía de Jesús el Pobre. Dicho vano está rasgando el muro original [A100] aprovechando una ventana igualmente original. También incluimos en este periodo las rozas para tubos eléctricos [A124] y el último enlucido [A125], que se aplica exclusivamente en el primer tramo.

Será en la segunda mitad del siglo XX cuando hagan aparición diferentes intervenciones de carácter restaurador. Según consta en los registros del Ministerio de Cultura hay una actuación de José Manuel González-Valcárcel (año 1965; signatura caja 71.171) y tres de Amparo Berlinches (dos en 1979 y una en 1980; signaturas cajas 77.055, 86.883, 80.953) de las que sólo la de González-Valcárcel afectó a la torre. Sabemos, no obstante, que Berlinches continuó actuando en ella a finales de los 80 .

La reparación contemporánea más antigua afectó al remate del machón central, por lo que su última planta aparece hecha de moderna fábrica de ladrillo con perfiles metálicos en las esquinas [A115]. Creemos que el machón original debió llegar a la misma o parecida altura que marca la restauración por lo que hemos de pensar que tuvo que ser desmontado parcialmente y saneado a causa de su mal estado de conservación.

Achacamos a la obra de González-Valcárcel las siguientes acciones: reparación de la escalera desde la puerta del coro hasta su remate [A171], parcheados en el interior [A118] y reparaciones en los muros exteriores de la torre [A169]. La nueva escalera es una obra mixta de cemento y madera que reutiliza bordes de escalón o atoques de una escalera anterior, que no sabemos si era la original u otra más tardía. La cota que lleva coincide con la que tendría la subida antigua.

La última intervención sufrida por la torre ha sido la dirigida por Berlinches en la década de los 80 [A137]. Su acción es visible tanto dentro como fuera. En la zona baja del exterior se coloca un forro de ladrillo, enfoscado con cemento. También se parchean algunas partes del muro, bien con ladrillo, bien con cemento patinado para fingir ladrillos. Al interior, los trabajos quedaron paralizados tras una primera fase destructiva que consistió en el picado de todas las capas de enfoscados que cubrían la fábrica antigua en la primera planta de escalera, tanto en el machón como en los muros exteriores, y en el desmonte de las escaleras modernas desde la puerta de entrada hasta la del coro. También se hicieron las trazas de una nueva escalera que nunca se llegó a realizar, pero, para recuperar su antigua anchura, se inició el relleno con ladrillo del corte que se había efectuado en el machón (fig. 7 y 8). Parece claro que el intento de Berlinches fue rehacer la escalera original en todos sus términos, llevándola hasta la cota de su subida primitiva, y para ello efectuó previamente el desmonte de la escalera moderna y la limpieza de las paredes, descubriendo los atoques originales cortados por la intervención moderna y con ello el trazado primitivo de la escalera. Desconocemos por qué interrumpió su obra antes de completarla. Otros hechos puntuales como el tabicado de las puertas de Cofradía y coro bien podrían inscribirse en estos esfuerzos.

Debido a la escasa actuación constructiva registrada en la torre, muchas actividades de menor rango, que no suponen en ningún caso cambios estructurales o remodelaciones, no se pueden asignar a una etapa concreta (SE en el listado final).

\section{LA SECUENCIA ESTRATIGRÁFICA Y CONSTRUCTIVA DE LA ETAPA I [A100]. UN MODELO CONSTRUCTIVO DESCONOCIDO}

El Método de Registro Estratigráfico permite obtener la secuencia constructiva, destructiva o restauradora, que se produjo sobre el edificio en estudio. Pero para llegar a este nivel, antes debemos pasar por una escala de análisis menor: nos referimos al estudio de la secuencia interna de cada actividad, a partir del análisis de los elementos que la componen (las UE de una A) o de elementos constructivos coetáneos, lo que suele permitir obtener su secuencia.

En el caso de la iglesia de San Pedro el Viejo, los elementos que forman parte de la fábrica original, secuenciados como etapa I [A100], presentan diferentes cuestiones estratigráficas y tipológicas que permiten corroborar su unidad. Pero además, a partir de su análisis podemos acercarnos a la puesta en obra y de esta forma recrear la técnica constructiva que utilizó. Debemos tener en cuenta que este proceso es para nosotros desconocido y sin paralelos, por lo que no deja de presentar cuestiones que habrán de someterse a revisión en el futuro. 


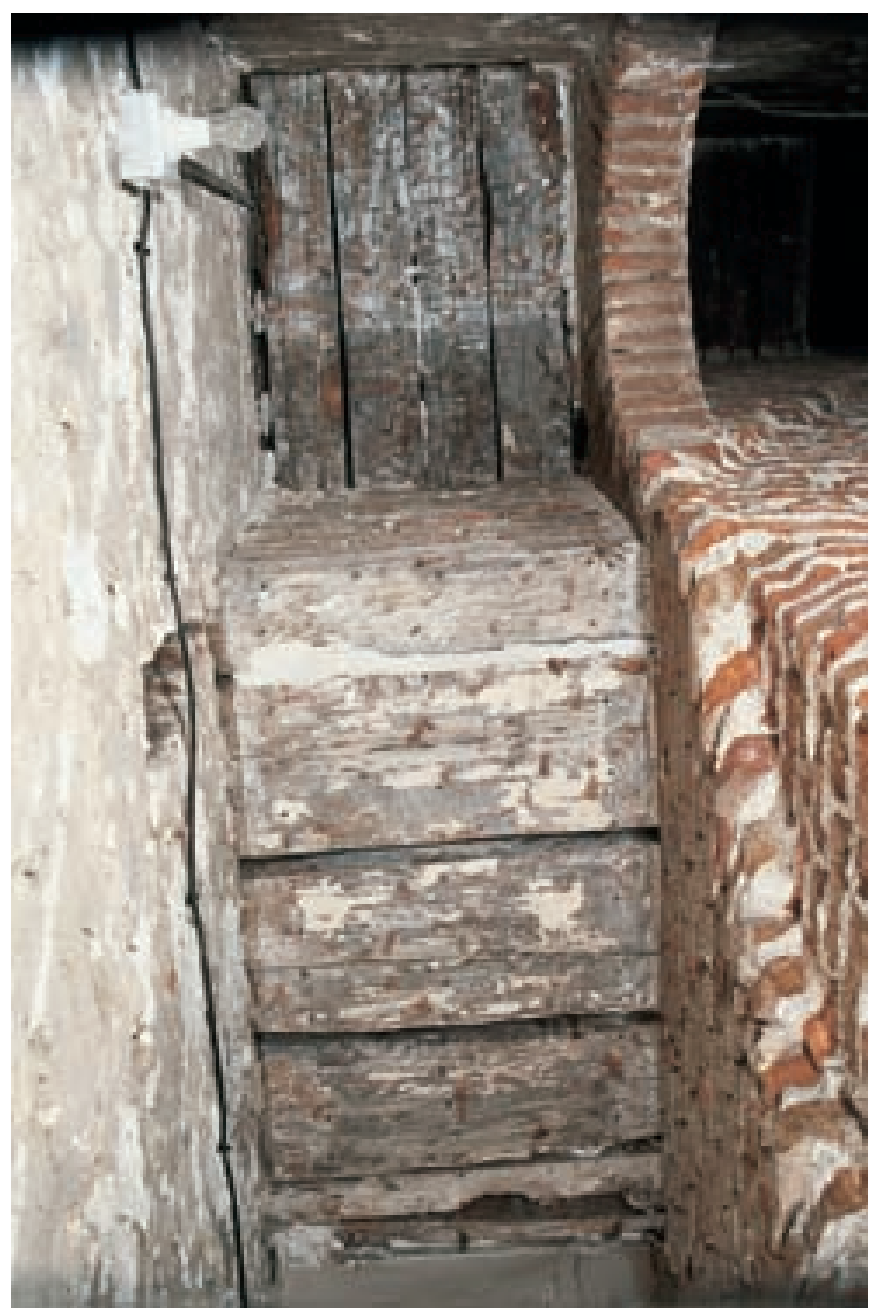

Fig. 5. Cubierta del primer tiro de la planta 1. a [A100]. A la izquierda el muro perimetral de calicanto con la ventana y a la derecha el muro de ladrillo del machón con el corte moderno [A105]

La torre de San Pedro, como ya hemos dicho, tiene cinco metros de lado y 30 de altura y está construida en el lado occidental de la iglesia, de modo que sobresalía medio cuerpo de su testero, coincidiendo con la arquería que separaba la nave central de la nave norte, quedando incluida en la nave central, aunque descentrada a su lado norte. Se compone de un muro perimetral de planta cuadrada y $1,25 \mathrm{~m}$ de grueso, que encierra un machón central de igual forma y $1,10 \mathrm{~m}$ de lado. Su fábrica es de ladrillo y calicanto, de modo que, en la parte baja del muro perimetral, queda el ladrillo visto en el exterior y el calicanto en el interior, mientras que en todo el machón y en el resto del muro perimetral interior, desde la tercera planta de escalera, la obra de ladrillo actúa como encofrado del relleno de calicanto. Entre el muro de fachada y el machón se desarrolla la escalera de cinco plantas y $0,75 \mathrm{~m}$ de ancho, asentada sobre mesetas (tres en cada tiro, una central y sendas para los rellanos comunes con los tiros vecinos) formadas con cargaderos de madera (madera reutili-

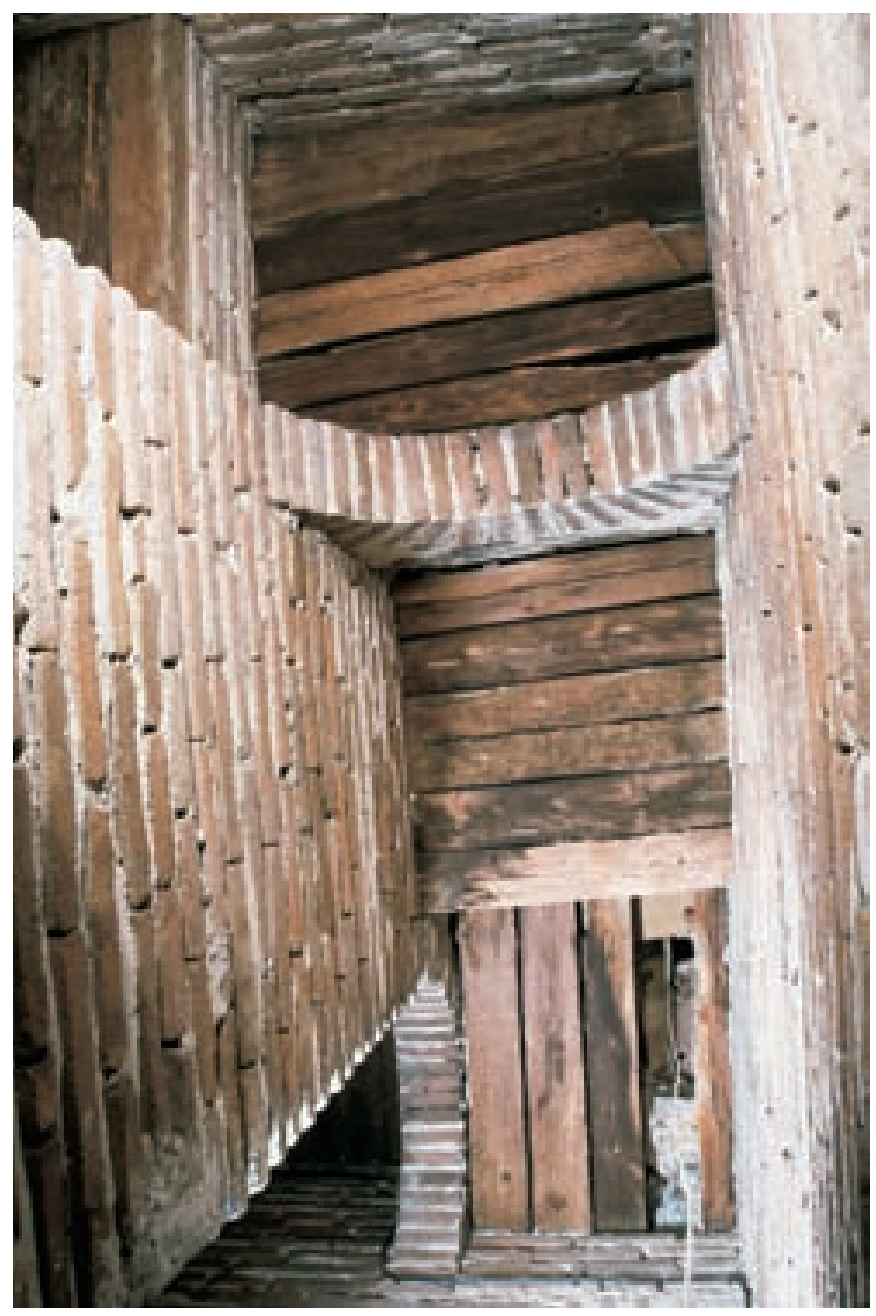

Fig. 6. Cubierta del tiro meridional de la planta $2 .^{\text {a }}$

zada de una obra anterior) que se sujetan en las esquinas en arcos de ladrillo (fig. 5, 6 y 12). Muretes de ladrillo, que atestan al muro y al machón, cierran los extremos de las mesetas y gradúan la inclinación de la escalera al variar su altura: de 18 a 16 hiladas en los primeros tiros; 9 a 6 en los siguientes hasta rebasar la segunda planta; y 4 a 2 en los pisos superiores. Efectivamente, la escalera tiene una característica especial, que en su recorrido inferior es mucho más pina (especialmente en el primer tiro, fig. 11) que en los superiores donde es muy plana (fig. 12).

Las observaciones que hacemos a continuación han sido posibles gracias a dos circunstancias ocurridas durante la vida de la torre. Una es la reforma de época moderna [A105] que desmontó la escalera original rebajando sensiblemente su nivel; y otra, el intento de reconstrucción de Berlinches [A137] que le obligó a desmontar la escalera moderna, descubriendo las estructuras de base sobre las que apoyaba la reconstruida, y a limpiar el enfoscado moderno 
descubriendo los pocos datos que quedaban del trazado original, especialmente restos de atoques embutidos en el machón y los muros perimetrales. Ambas circunstancias permiten que hoy comprobemos cómo se construyó la torre original, ya que de otro modo desconoceríamos este proceso, permaneciendo ocultos muchos detalles que han dado las claves para su comprensión constructivo/cronológica.

Pero no debemos engañarnos. Estas agresivas intervenciones no pretendían una documentación histórica y con ellas se provocó la ocultación y la desaparición de intervenciones positivas anteriores. Por ejemplo, el desmonte de la escalera moderna, el forro de ladrillo y el enfoscado de cemento con los que Berlinches pretendía reconstruir la escalera original nos impiden conocer la superficie del corte de la escalera efectuado en época moderna.

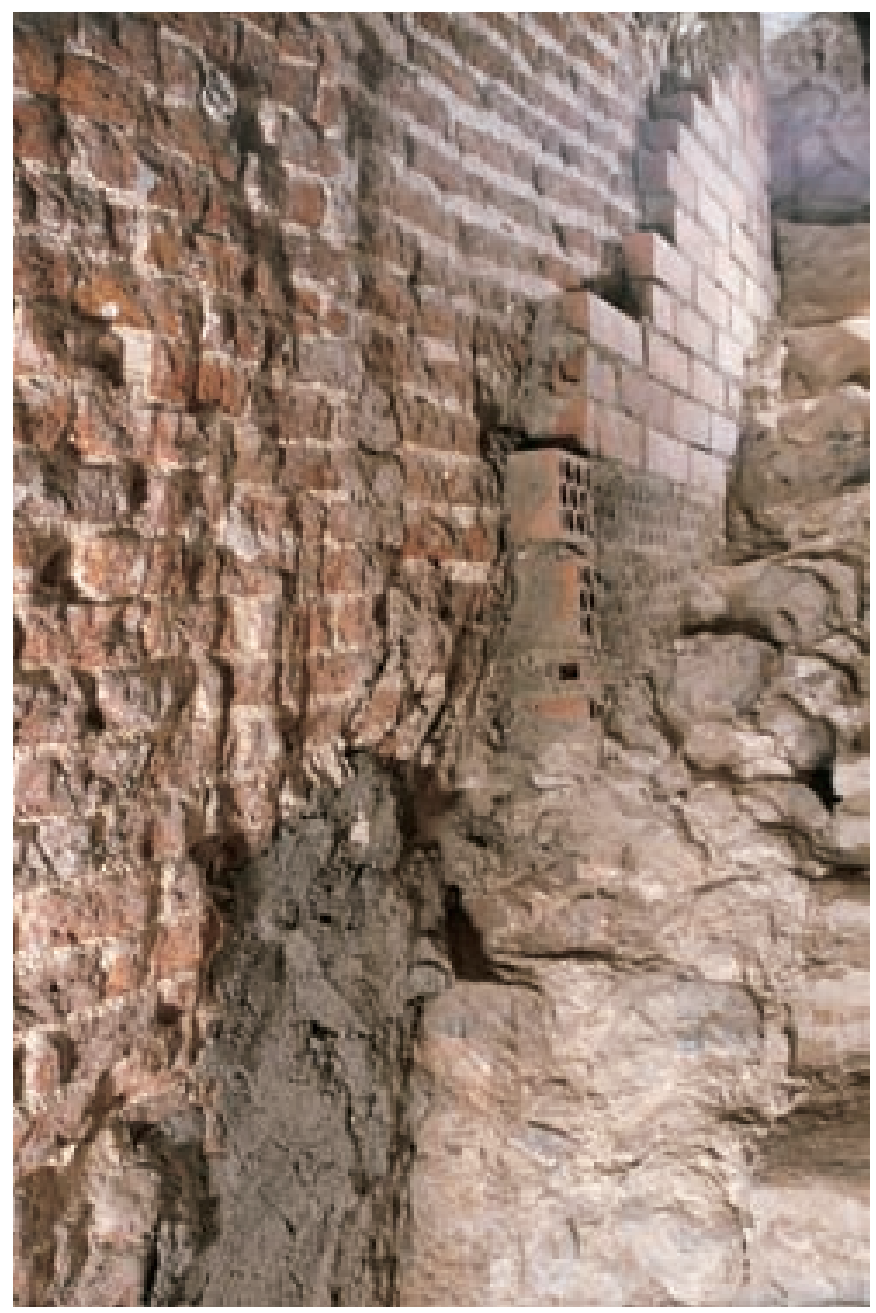

Fig. 7. La pared del machón central desde la puerta de entrada, planta 1. ${ }^{a}$ cara E. En primer término la reforma de la jamba de entrada [A101 UE1003]. A la izquierda, el muro de ladrillo del machón [A100] con el corte de época moderna [A105 UE 1001]. A la derecha el muro de ladrillo y barro [A100 UE1087] y el cimiento de calicanto [A100]. Entre ambos la intervención de A. Berlinches [A137]

\section{El proceso histórico/constructivo}

Fase I A. El cimiento. En realidad desconocemos cómo era el cimiento de la torre. Gracias al hormigón de cal que se ve en el espacio libre de la puerta (al haberse arrancado los umbrales primitivo y moderno), lo podemos suponer como un gran bloque de calicanto que ocuparía toda la superficie cuadrada de la torre.

Fase I B. El muro de ladrillos unidos con barro (fig. 13). Una vez construido el cimiento se realizó un muro de ladrillos, unidos con barro y trabados con madera, que iba a servir como encofrado del calicanto (fig. 7 y 8 [A100 UE1087]). La presencia de un enfoscado o enlucido en todas sus caras confirma la construcción previa de éste y la posterior del núcleo de calicanto del machón, a su in-

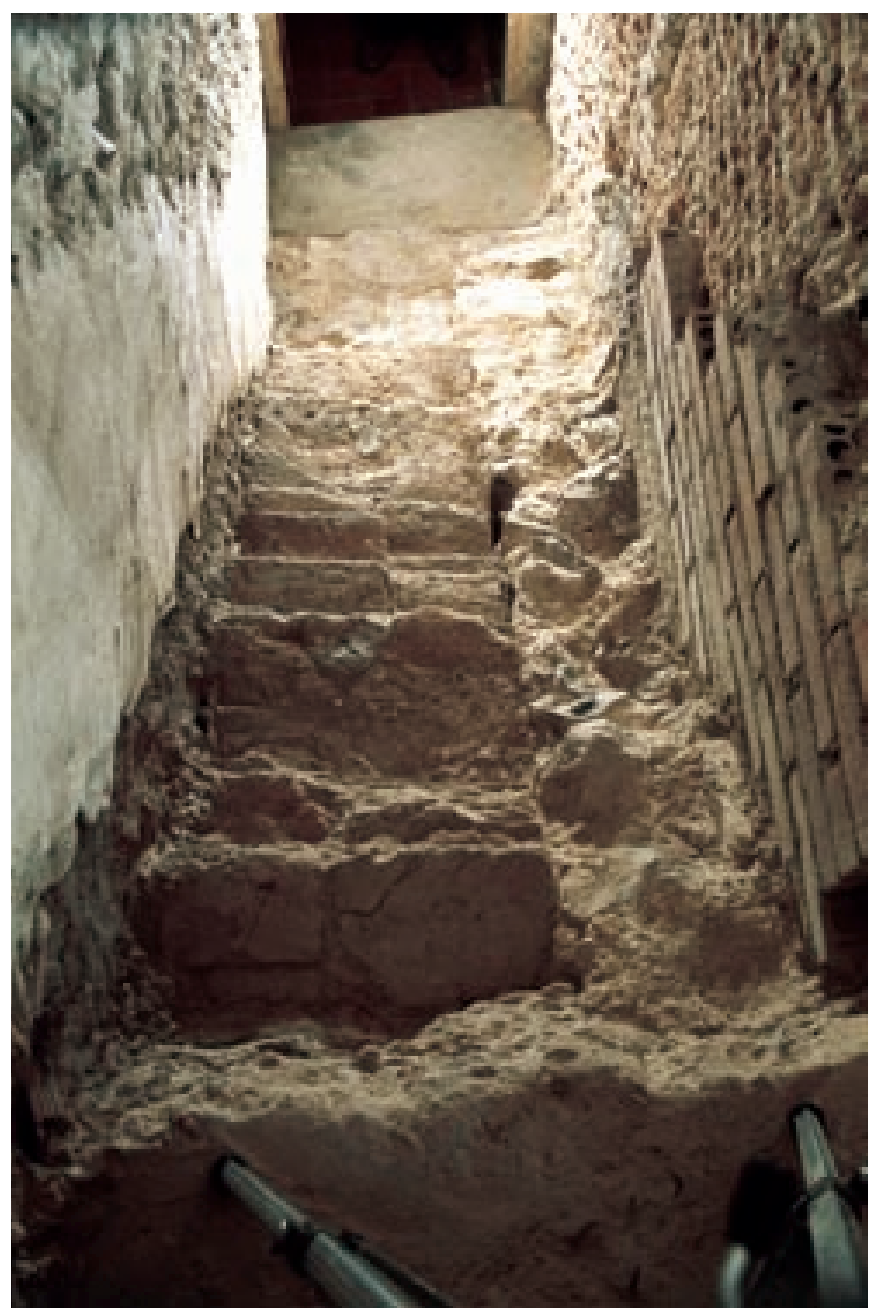

Fig. 8. Primer tiro, oriental, de la planta 1. ${ }^{a}$, bajando. En el centro el muro de barro [A100 UE1087] y el cimiento de calicanto del machón [A100].

Inmediatamente a izquierda y derecha, la restauración de A. Berlinches [A137]. A continuación la obra original [A100], a la izquierda el muro perimetral de calicanto y a la derecha el muro de ladrillo del machón cortado en época moderna [A105] 
terior, y del muro de calicanto perimetral, a su exterior. $\mathrm{Al}$ enlucir sus caras se consigue una superficie llana para impedir el contacto entre el ladrillo y el calicanto, porque, de no hacerlo así, el hormigón al fraguar «engancharía» en las juntas de ladrillo complicando su desmonte posterior. Enseguida veremos cómo, sin embargo, el fraguado incompleto de este enlucido provocó un efecto no deseado.

Para la comprensión del procedimiento constructivo de la torre se hace imprescindible el análisis detallado de este muro. Se desarrolla en altura en tres lados (este, norte y oeste) de la primera vuelta de la escalera y hoy presenta la forma de una estructura aparentemente escalonada. Su grueso es de entre 53 y $55 \mathrm{~cm}$ y sus ladrillos miden $30 / 31 \times 21 \times 4,5 / 5 \mathrm{~cm}$, presentando un aspecto diferente a la de los restantes ladrillos de la torre, de color más ocre y menos rojo, por haber sufrido una cocción menos fuerte.

Parece formar una estructura en «U», alargada en la dirección N-S, que envuelve al machón central justamente en los tres primeros tiros donde éste también es de planta rectangular. Además, ocupa los tiros donde la escalera aún no volaba y por lo cual tenía que apoyar sobre una estructura de base cuya función creemos cumplió este mismo muro de ladrillo y barro. Evidentemente hoy no se conserva hasta esa altura en todo su desarrollo pues hubo que desmantelarlo para disponer la escalera sobre el, pero deducimos que subió hasta donde comienza a volar la escalera porque coincide con la altura de la huella que el enfoscado de sus caras exteriores y sus propios ladrillos han dejado "pegada" en las caras internas de calicanto de los muros perimetrales (fig. 9). Esta huella se formó debido al mayor agarre del mortero del calicanto que «tiró» de su enfoscado y de la primera capa de sus ladrillos, lo que indica que posiblemente estaba aún fresco cuando esto ocurrió. Actualmente se le adosa por todos sus lados el calicanto, tanto del muro perimetral como del cimiento del machón central, de modo que se puede suponer que actuó como su encofrado en los dichos tres tiros.

De este modo consideramos que el muro de ladrillo y barro cumplía una doble función, la de encofrado del calicanto, durante la obra, y la posterior de apoyo de los tiros de la escalera que no iban volados. La perfecta adecuación de sus características a las dimensiones, forma y finalidad de las distintas partes de la torre, así como su pobre construcción, que hace pensar en una obra provisional, obligan a ponerle en relación con el proceso de obra como estamos haciendo y a rechazar la posibilidad de que se tratara de un resto constructivo anterior que reutilizara la nueva obra.

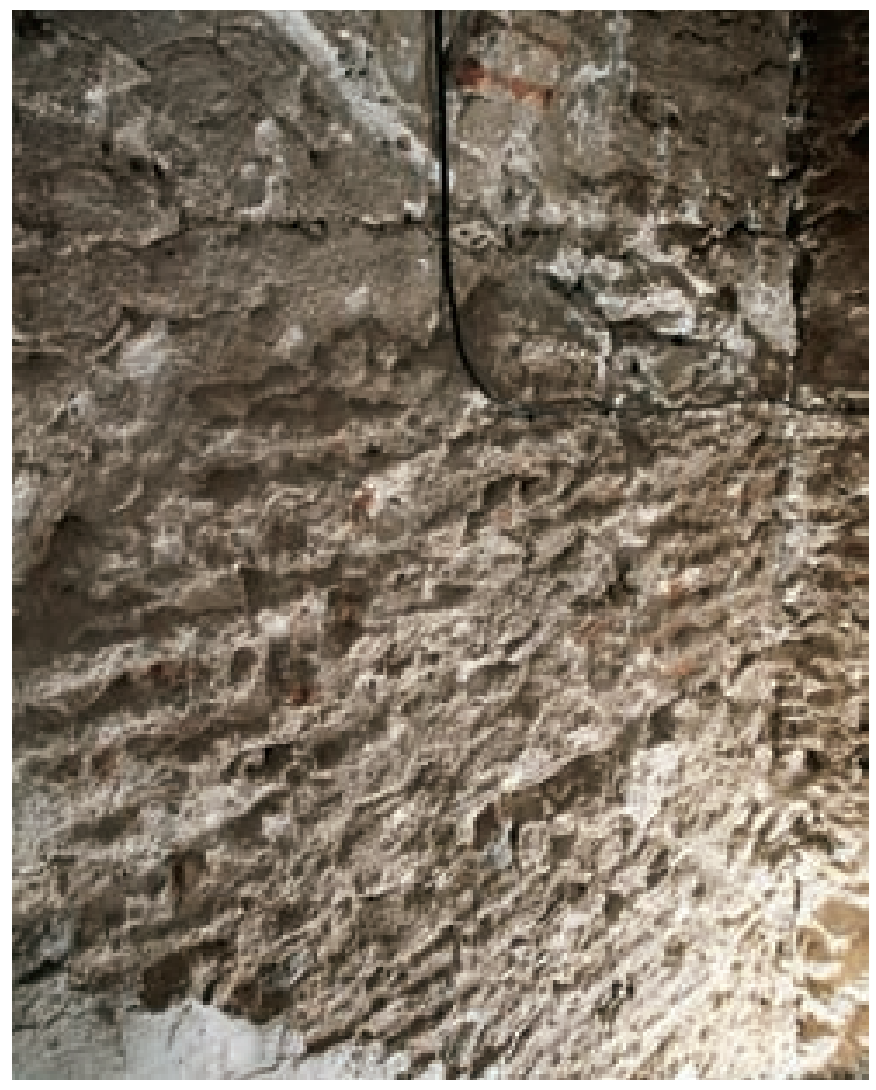

Fig. 9. Huella del muro de ladrillo y barro, utilizado como encofrado del muro perimetral de calicanto en cuya superficie se conserva [A100 UE 1022]. Planta 1.a, muro $\mathrm{N}$

Fase I C. El muro de calicanto perimetral y la fachada de ladrillo visto. El muro de ladrillo y barro es anterior a la obra de calicanto del perímetro exterior, y por lo tanto a la fachada de ladrillo, y a la del machón. Luego se alzarían hasta la altura que habían de tomar los tres primeros tiros de la escalera el forro de ladrillo de las fachadas exteriores y el núcleo de calicanto perimetral vertido en el encofrado que le ofrecían ambos muros, el muro de barro por dentro y las fachadas de ladrillo por el exterior. Al ir alzándolo por tongadas, que se dejaría que fraguaran antes de continuar, no había peligro de que la presión del calicanto derribara el muro de ladrillo y barro.

El remate del lado sur es el que queda más inconcreto por falta de datos. El problema principal es la unión entre los extremos meridionales (especialmente el más occidental) del muro de barro y de la fachada de ladrillo de modo que cerraran funcionalmente el encofrado del calicanto, tal, por ejemplo, como lo hemos dibujado de modo sencillo en la fig. 13. Pero es más probable que la fachada de ladrillo también se elevara por completo en el lado sur, a la vez que el resto de obra, pues no encontramos en ella soluciones de continuidad que hagan pensar en que se dejara una abertu- 
ra coincidente con el grueso del machón. También pudo ocurrir que esta supuesta abertura coincidiera con el muro de testero occidental de la iglesia, continuando el eje N-S de la torre como define el muro de testero en el lado norte (fig. 16). Por otra parte, está el marco de la puerta de entrada, con jambas de ladrillo y desconocido cargadero de madera, que también pudo construirse o en un momento posterior, cuando se levantó el machón central, o en este momento, antes de iniciar esta obra.

Fase I D. Desmonte del muro de ladrillo con barro (fig. 16). Una vez alcanzada la altura de los tres primeros tramos, se desmantelaría el muro de ladrillo y barro formando una rampa que seguiría el trazado que había de tener la escalera definitiva.

Fase I E. El machón de calicanto y ladrillo. El cimiento de calicanto del machón central sube en caracol (fig. 7 y 8), siguiendo la rampa del muro de ladrillo y barro una vez desmontado, y debe extenderse, tongada a tongada, hasta el muro de ladrillo de la fachada sur. De este modo el cimiento del machón es $40 \mathrm{~cm}$ más ancho que el propio machón en su alzado de ladrillo, exactamente los $20 \mathrm{~cm}$ de diferencia que hay a cada lado entre los 0,75 del ancho del tiro de escalera y los 0,55 del murete de ladrillo.

La escalera original, hoy desaparecida, era coetánea al machón de ladrillo, como demuestra que los restos de atoques de madera de sus peldaños forman unidad con el calicanto del machón allí donde falta su forro de ladrillo cortado en época moderna. Este es otro argumento que obliga a pensar que el machón se hace una vez desmontado el muro de ladrillo y barro, el cual adquiere su aspecto escalonado en este momento, para montar la escalera definitiva sobre él.

De acuerdo con lo comentado anteriormente, no conseguimos una solución adecuada para el cierre de la obra por el lado sur, de modo que el calicanto líquido no vertiera al exterior, pues, igual que pensamos que el muro sur ya pudo estar construido, también creemos que el espacio del futuro machón y su prolongación hasta el muro sur debía estar vacío y que su relleno tuvo que efectuarse ahora, coetáneamente a la construcción de los primeros tramos de la escalera.

Fase I F. Continuación de la obra. Por encima de estos tres primeros tramos, la torre se construye elevando simultáneamente el machón de ladrillo relleno de calicanto, la escalera volada y el muro perimetral de calicanto. En el cuarto tiro no existen restos de la estructura de ladrillo y barro que se hace imposible a partir del quinto por la in-

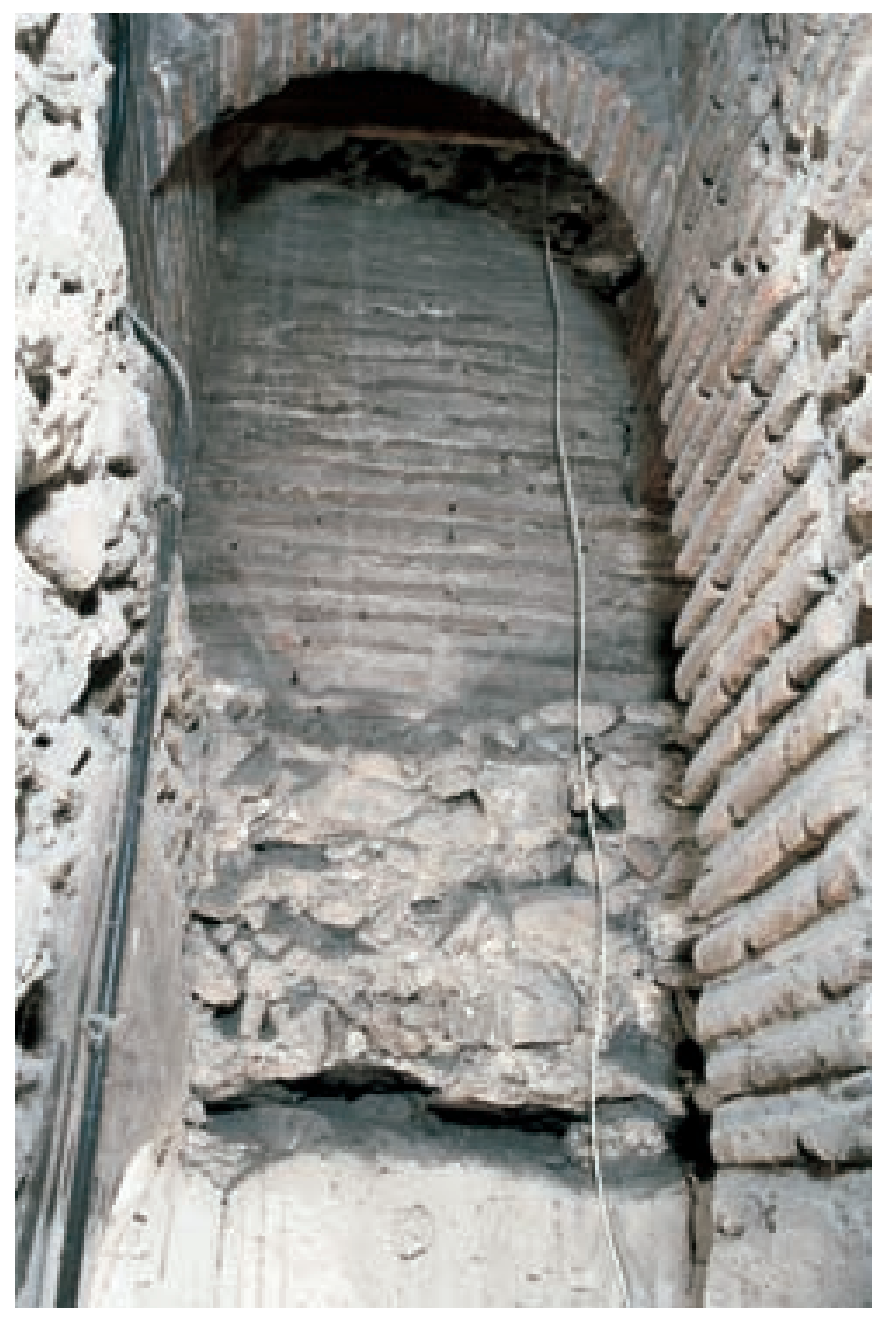

Fig. 10. Paso de la fábrica de calicanto a la de ladrillo [A100]. Planta 1.a, muro S

mediata presencia de las mesetas y los arcos de apoyo para los tiros superiores, donde la escalera ya es volada. Por otra parte, el muro perimetral mantiene su estructura de ladrillo en fachada y calicanto interior hasta completar la segunda planta. Ahora se construye empleando un encofrado de madera del que se observan las huellas por encima de donde se conservan las del enfoscado del muro de barro ya citadas. El machón central toma su forma cuadrada y utiliza como encofrado en sus cuatro lados el muro de ladrillo visto.

Fase I G. Sustitución de la obra de calicanto por la de ladrillo (fig. 4, 10 y 15). Al llegar al arranque de la tercera planta, se sustituye en la cara interior del muro perimetral el calicanto por el muro de ladrillo visto, ligeramente retranqueado en su arranque y escalonando el remate del calicanto, machihembrando quizás con la intención de mejorar el agarre entre las dos obras. 


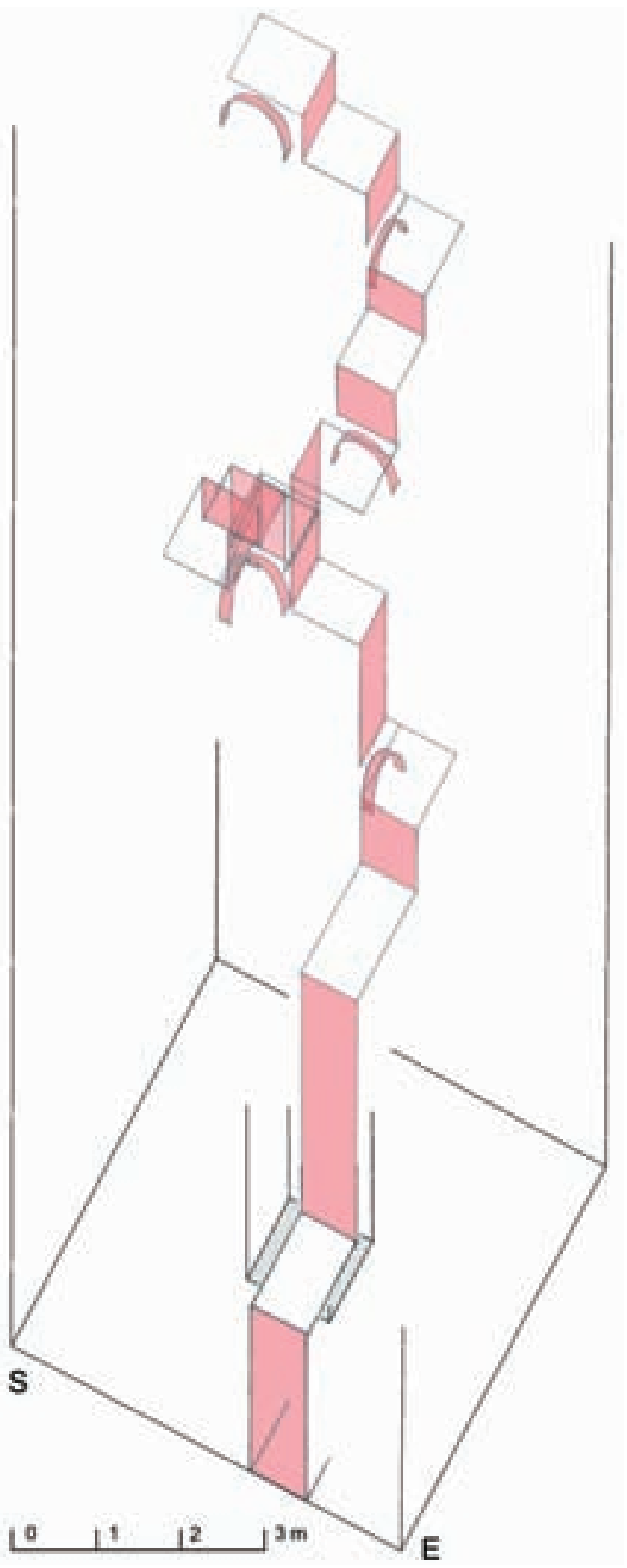

Fig. 11. Esquema en perspectiva de las mesetas de la primera planta y dos tiros de la segunda en la que se observa la fuerte pendiente de esta parte de la escalera

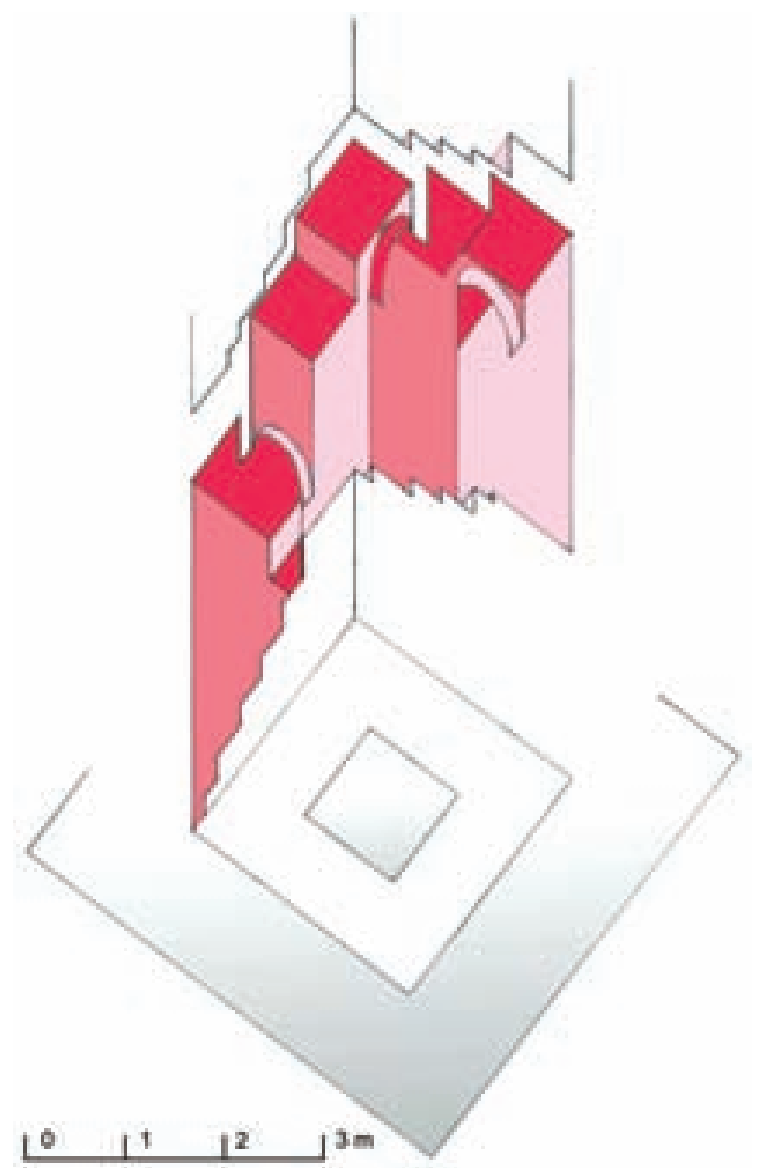

Fig. 12. Esquema de la escalera en los tiros E y $\mathrm{N}$ de la planta cuarta, con tendencia a una mayor horizontalidad

Igual que no se puede suponer que el muro de ladrillo y barro pertenezca a una obra anterior, tampoco se puede suponer de la obra de calicanto vista del muro perimetral en las dos plantas inferiores. Fundamentalmente por su íntima unión y semejantes funciones y características que el resto de obra de ladrillo y calicanto en todo el desarrollo de la torre, en cualquier lado que se pueda comprobar, bien sea a cara vista o como relleno, tanto en el machón central como en el muro perimetral.

\section{OTRAS CUESTIONES CONSTRUCTIVAS}

La unión entre torre e iglesia (fig. 17 y 18). En la cara norte de la torre se puede observar su unión con el arranque del último arco de la arcada de la iglesia. La observación es muy parcial, limitada por la presencia de elementos constructivos y enfoscados que impiden comprobar las características de los muros que nos interesan. Por otra parte, en el desván de la casa adosada al Norte de la iglesia se puede observar la cara exterior de su muro de cierre, construido con verdugadas y cadenas de ladrillo (de similares dimensiones a los de la torre) y cajones de tapial (fig. 18). Podemos ase- 


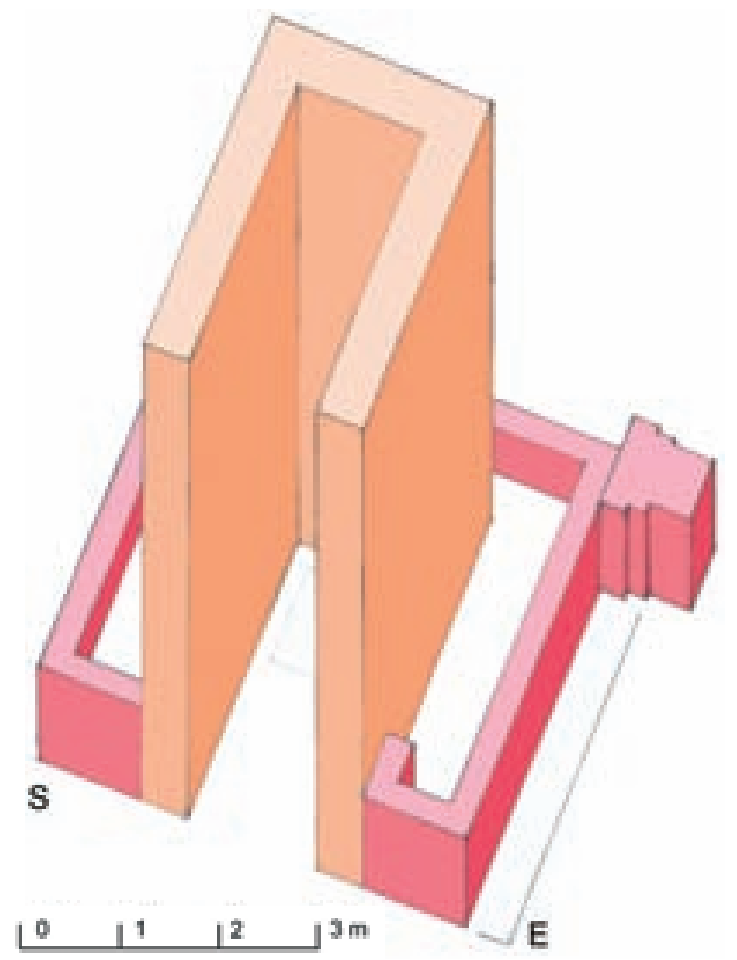

Fig. 13. Esquema en perspectiva de la construcción de muro de ladrillo y barro (en marrón) e inicio de la fachada de ladrillo visto (en rojo)

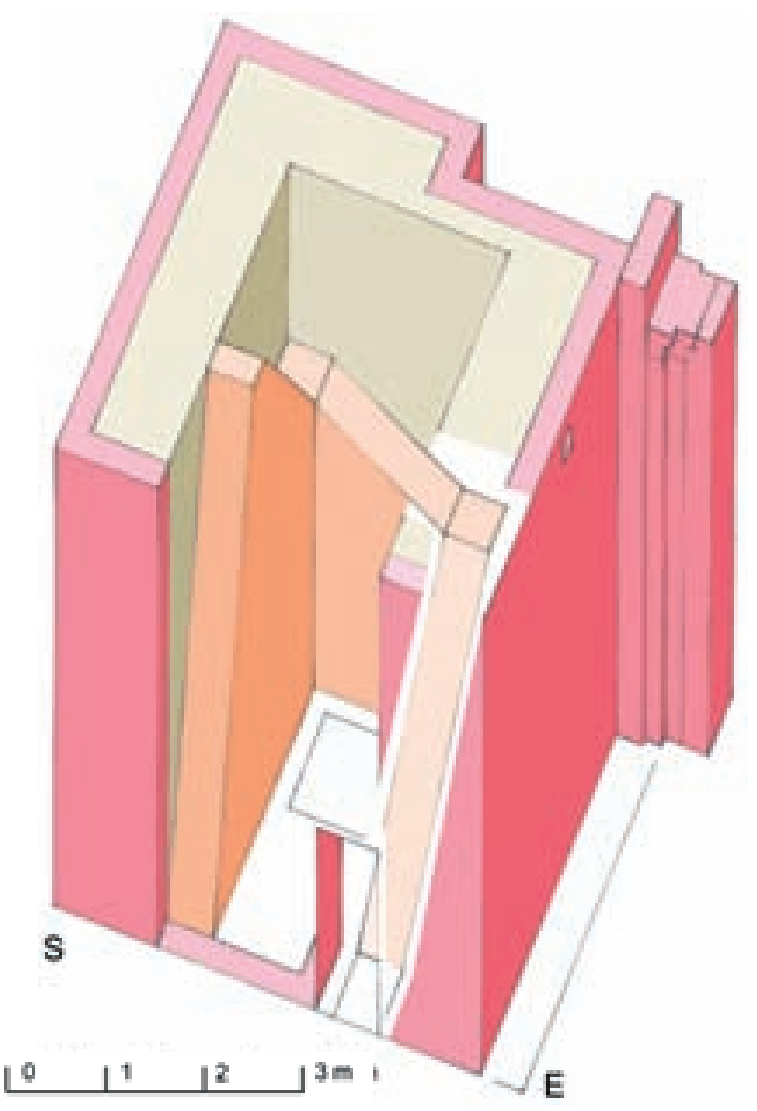

Fig. 14. Esquema en perspectiva del corte del muro de barro, una vez efectuada la construcción del muro perimetral (en gris, la obra de calicanto)

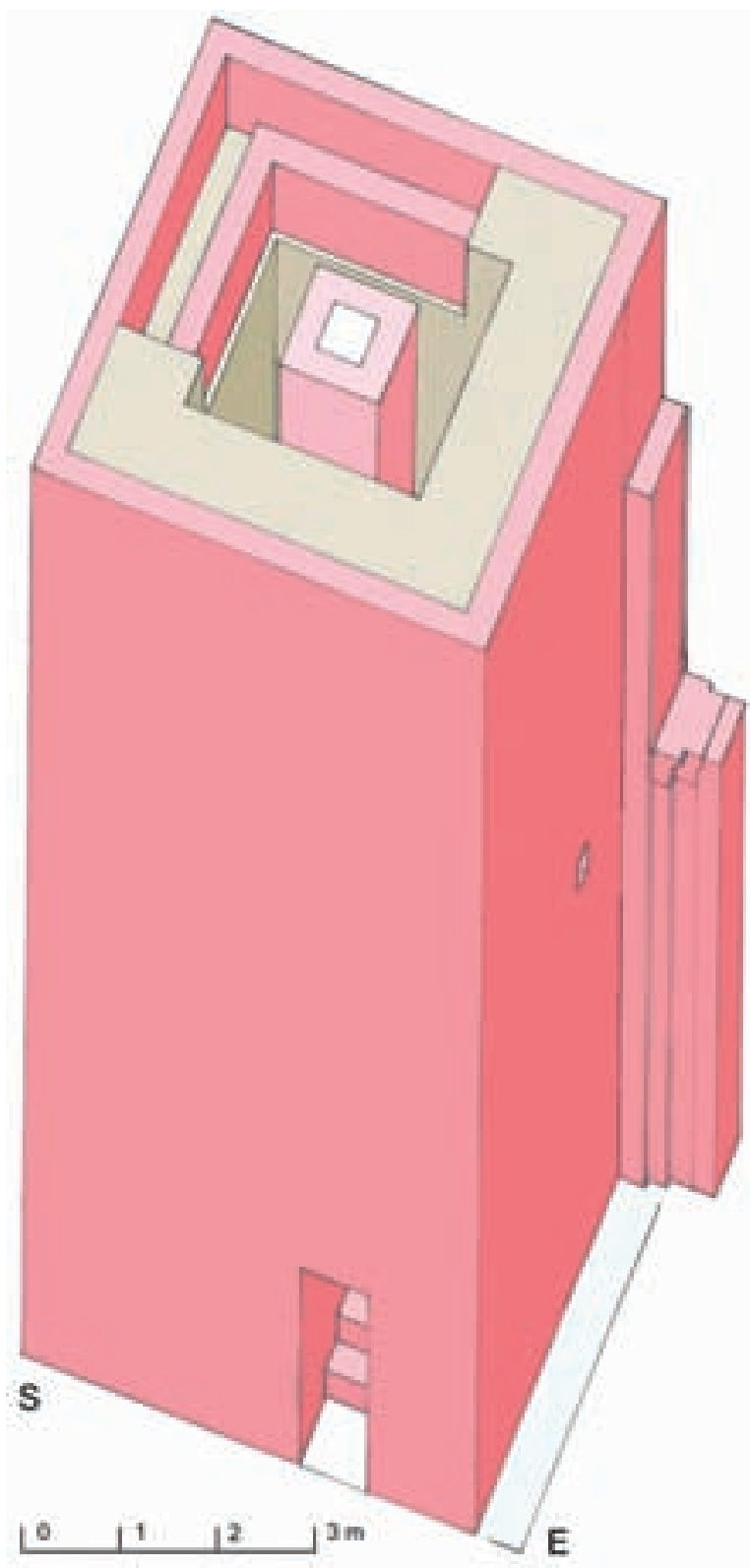

Fig. 15. Esquema en perspectiva del final del muro de calicanto interior y arranque del de ladrillo, a la altura de la planta tercera

gurar con suficiente certeza que este muro gira y remata en el eje N-S de la torre como testero occidental de la iglesia (fig. 11 y 16) ${ }^{5}$. Entre él y el arranque del último arco de la iglesia, la fachada norte de la torre forma otro arco sencillo, cuyo asiento se refuerza con un muro en resalte y cuyo intradós debía estar situado a la misma altura que el intradós

5 Frente a la opinión de Abad, 1991: 2, fig. 18 que, quizás por no haber podido comprobar esta zona tras la intervención de Berlinches, supone que la torre quedaba fuera de la iglesia y adosada a su testero (comparar con nuestra fig. 16). 


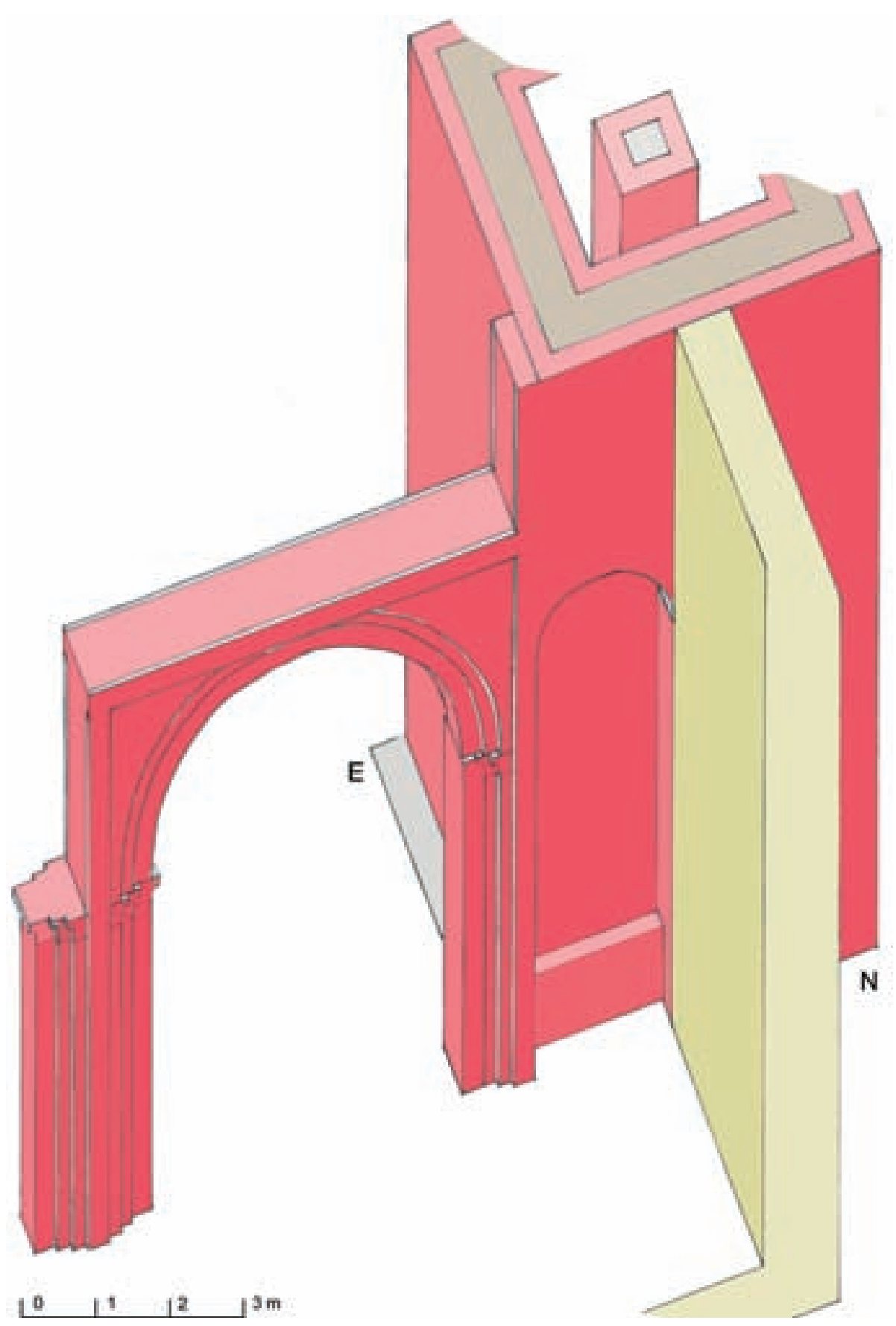

Fig. 16. Esquema en perspectiva de la unión entre la torre y la iglesia, visto desde Norte (en verde, los muros de la iglesia)

del último $\operatorname{arco}^{6}$. Ambas obras se unen entre sí alternando sus enjarjes de modo que facilitan la autonomía de sus partes. Así el muro con el arco de la torre es semindependiente del aparente relleno de su fondo que se ha podido mover

6 Según la pilastra enjarjada a la torre, los pilares de la iglesia serían cruciformes con dos codillos, como ya propone Abad, 1991: 1, 202-204. Los arcos son de medio punto (frente a la norma general toledana de herradura) y recuadrados, Id. 1, 206, 210. sin arrastrarlo. Una estructura similar (salvo la ausencia de la arcada) debía presentar la cara sur de la torre, aunque es difícil de comprobar por la presencia de los locales de la Cofradía de Jesús el Pobre.

Andamios y mechinales. La torre no presenta mechinales constructivos, salvo las excepciones de los paños orientales de los muros exteriores norte y sur, en el relleno de los arcos que acabamos de citar y que debieron servir para volar andamios desde los que armar sus vueltas y para sujetar 


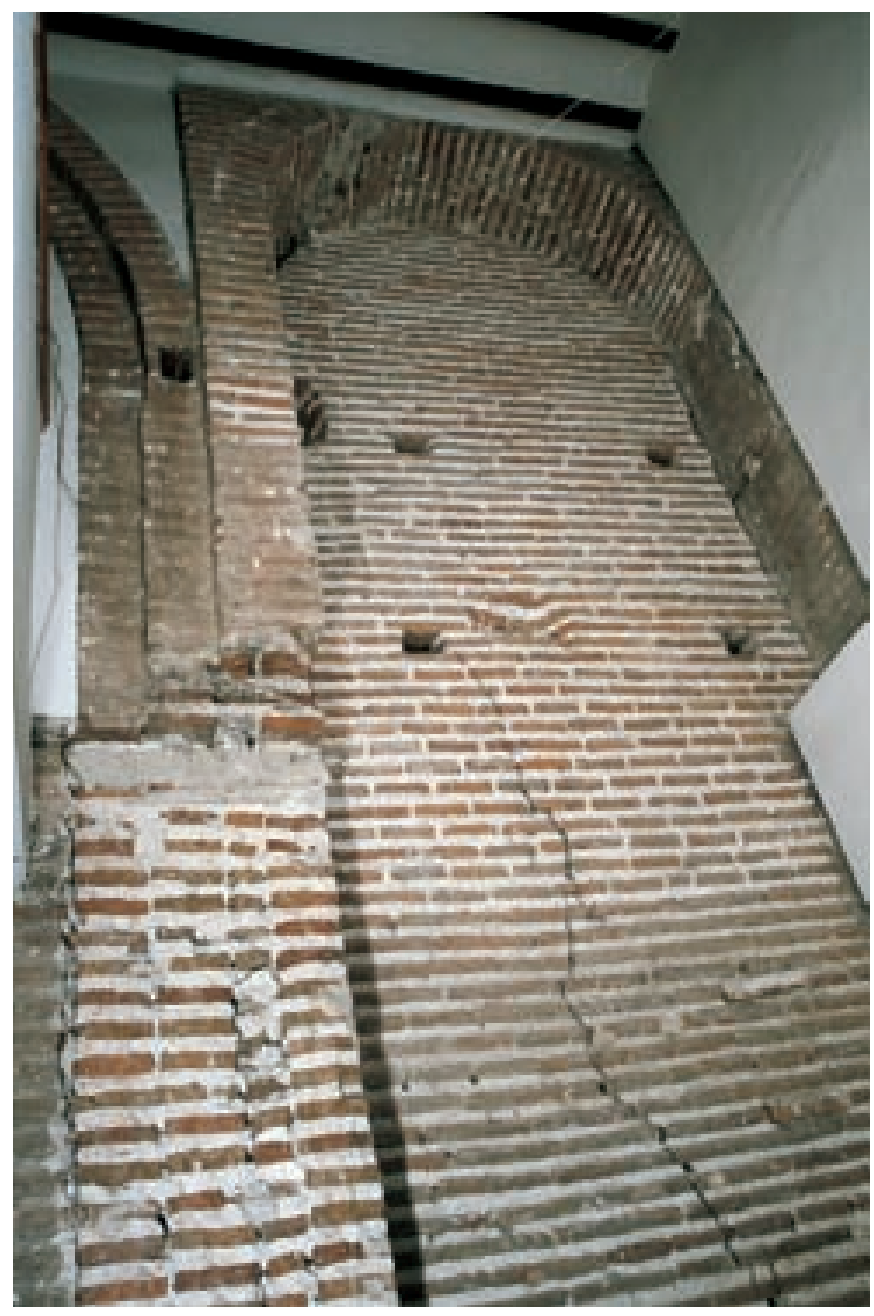

Fig. 17. Unión entre la torre y la arcada N de la iglesia. Muro N exterior [A100]. El muro de la derecha es el supuesto testero occidental de la iglesia

sus cimbras. La construcción de la primera planta se pudo apoyar en andamios exteriores sin alma, pero también pudo edificarse sujetando los andamios en el propio muro de ladrillo y barro, ocupando el hueco interior donde luego iba a elevarse el machón. La ausencia de mechinales en el alzado de la torre afianza la hipótesis de que el machón, la escalera volada y el muro perimetral se construyeron desde el interior, tomando como base la propia escalera, sobre la cual se montaría el encofrado de la planta segunda y el ligero andamio necesario para los demás.

La cuestión mensiocronológica de los ladrillos y su aparejo. Las dimensiones de ladrillos y sus juntas son constantes en todo el alzado, aunque aceptando amplias variaciones y tendiendo a ser de menor dimensión cuanto más alta es la obra, tanto en el interior como en el exterior. Estas variaciones se demuestran constantes y no bruscas por lo que no pueden achacarse a obras o momentos distintos, sino, probablemente, a una selección intencionada del material. Los

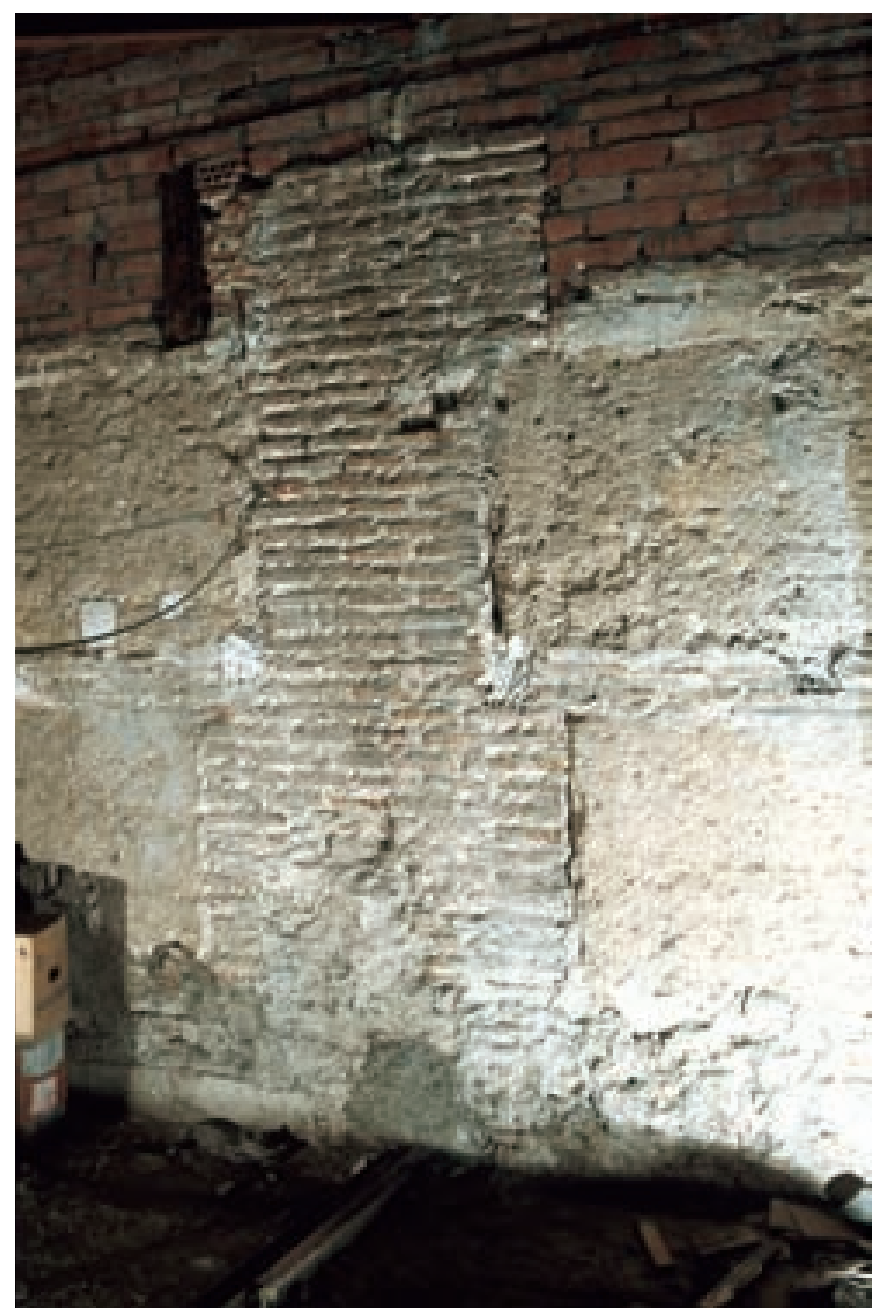

Fig. 18. Cara exterior del muro $\mathrm{N}$ de la iglesia, de verdugada y tapial [A100 UE1094]

ladrillos miden $27,5 / 30 \mathrm{~cm}$ en su lado mayor, $17,5 / 18 \mathrm{~cm}$ en el menor y $3,5 / 4 \mathrm{~cm}$ de altura; con variaciones de $27 / 34 \mathrm{~cm}$ en su lado mayor, $17 / 21$ en el lado menor y $3 / 5,5$ en la altura. La proporción ronda los $2 / 3$ o poco menos. Las variaciones máximas harían pensar en la existencia de un módulo distinto de $32 / 34 \times 20 / 21 \times 5,5 \mathrm{~cm}$ que sólo hemos observado en las caras exteriores tanto sur como oeste, pero no de modo sistemático sino alternando con el módulo común mayoritario ${ }^{7}$. En éste parece observarse una tendencia decreciente en la media que va de los $30 \times 18 \times 4$ en el exterior; y en el interior $29 \times 18 \times 4$ en la planta segunda, $28 \times 17,5 \times 3,5$ en la tercera y $27,5 \times 17,5 \times 3,5 \mathrm{~cm}$ en la cuarta. Llama la atención la amplia variación en la medida de los ladrillos, más si añadimos la de los ladrillos del muro

7 Las dimensiones entran en la proporción de 2/3 que, según Abad, es la normal del área toledana y con su observación de que las dimensiones mayores corresponden a las fábricas más modernas (1991: I, 228-229). 
de barro $(30 / 31 \times 21 \times 4,5 / 5 \mathrm{~cm})$, de proporción cercana a la de los ya vistos aunque desviada en sentido contrario. Esta variabilidad hace pensar en la inexistencia de una norma administrativa que rigiera su relación tamaño/precio.

Las juntas horizontales varían entre 3 y $4 \mathrm{~cm}$ con márgenes hasta los 2,5 y $5,5 \mathrm{~cm}$, mientras que las verticales o se efectúan a ras o miden $1 \mathrm{~cm}$ como máximo. Las horizontales rematan con doble bisel, único bisel hacia arriba o hacia abajo o a ras, variaciones que individualizan las diferentes manos que participan en la obra. La de doble bisel parece que se utilizó sistemáticamente en la iglesia.

Normalmente el aparejo de los ladrillos se ordena en grupos de hiladas que alternan a soga y tizón, sin una regla determinada pues pueden agrupar entre dos y diez o aún más hiladas. Esta norma pretende formar jarjas internas para agarrar perfectamente en el calicanto. Las esquinas se suelen resolver mediante el enjarje de los ladrillos, pero tampoco esto es general, pues es normal la alternancia de tramos de la misma esquina con uniones resueltas a testa y otros resueltos a enjarje, de modo que, si nos fijamos respectivamente en uno u otro tramo despreciando los vecinos, desconcierta la sensación de que la obra pertenece a la vez a momentos distintos y a momentos coetáneos. También se identifican bancos constructivos que alcanzan, normalmente alrededor de $80 \mathrm{~cm}$ de altura, pero con variaciones que pueden quedarse en los 65 , según la situación, y cuyas características de altura de hiladas, tipo de junta y dimensión de los ladrillos es el indicador más claro del cambio de cuadrilla de trabajo. Entre los bancos suelen aparecer soluciones de continuidad verticales. De este modo parece quererse evitar juntas continuas horizontales que pudieran devenir en el futuro en deslizamientos.

Los morteros. Según los análisis efectuados (Guarás 2002), son homogéneos, todos ellos de cal, con excepción de uno de yeso. Los áridos son los propios de la cuenca del Manzanares en que se ubica Madrid, con la inclusión en algunos de ladrillo procedente de la misma obra. Su mayor o menor calidad, graso o magro, áridos más o menos finos, puede achacarse a variaciones casuales de su fabricación o a la función para que se requirieron. Algunos morteros son considerados «idénticos» por la analista: un primer grupo engloba los procedentes del cimiento de calicanto del machón central de la primera planta [A100 UE1086] y dos del muro de ladrillo del paño oriental exterior norte [A100 UE1000], a los que se puede unir otro del muro de calicanto perimetral interior también de la primera planta [A100 UE1000]; y otro segundo grupo con los procedentes del muro de ladrillo de la segunda, muro $\mathrm{S}$, y del arco de la tercera, muro $\mathrm{N}$ [A100 UE1000]. La identidad de estos morteros, aún sin llegar a considerarlos estrictamente de «un mismo momento de obra» como pretende la analista (aunque de hecho los del primer grupo proceden de una misma altura de obra que constructivamente, como vimos, debió efectuarse casi inmediata), es otro indicio de la unidad de la obra, ya sea exterior o interior, cimiento o muro, obra de calicanto o ladrillo. Los dos que completan el grupo homogéneo proceden uno del pilar de ladrillo con que arranca la arquería de la iglesia, cuidado, graso y fino [A100 UE1000], y otro de la huella del muro de ladrillo y barro sobre la superficie del muro $\mathrm{N}$ de calicanto de la primera planta [A100 UE1022], cercano a los del segundo grupo citado. Las excepciones están representadas por una muestra del enfoscado del muro de barro, casi cal pura, sin áridos [A100 UE1087]; y el de reparación del hueco preparado para apoyar los maderos de la primera meseta, único detectado de yeso [A100 UE1000].

\section{CONCLUSIONES}

Con esta secuencia constructiva del comienzo de la torre damos a conocer un sistema constructivo desconocido en nuestro ámbito científico a tener en cuenta en los estudios de la numerosa nómina de fábricas con estas características.

Se confirma la inexistencia de una construcción anterior a la torre. Tras el cimiento de la torre, la estructura de ladrillo y barro es el primer elemento construido, sin que por su condición endeble y efímera, de más de 10 metros de altura por $55 \mathrm{~cm}$ de ancho, de solo tres lados y construida con barro y enfoscada en sus caras y testas pudiera tener otra utilidad, ni como torre civil o religiosa. Su existencia se debe únicamente a su función de encofrado del núcleo de calicanto y del muro perimetral exterior de la torre y de soporte de los primeros tramos de la escalera. Lo mismo ocurre con el muro de calicanto perimetral visible en el interior de las dos primeras plantas que pertenece a la fábrica unitaria de la torre. La forma, composición, íntima conexión con los demás elementos y relación con las distintas características de la torre lo confirman para ambos elementos. Las fábricas de ladrillo y de hormigón de calicanto son coetáneas y dependen entre sí, de modo que la segunda actúa de cimiento y núcleo de la primera quien, a su vez, funciona como su forro y encofrado.

La torre es unitaria en todo su desarrollo y es igualmente unitaria con los pocos indicios que tenemos de la iglesia mudéjar y que se relacionan con ella. Conocemos abundantes datos sobre su proceso constructivo, aunque su condición de únicos y su conocimiento incompleto plantean cuestiones sobre su exacto desarrollo. A nuestro parecer, se debe aceptar este conocimiento incompleto y no se debe tratar de explicar acudiendo a otras justificaciones que no estén apoyadas por datos, como a la existencia de restos de edificaciones anteriores o pertenecientes a etapas cronológicas distintas. 


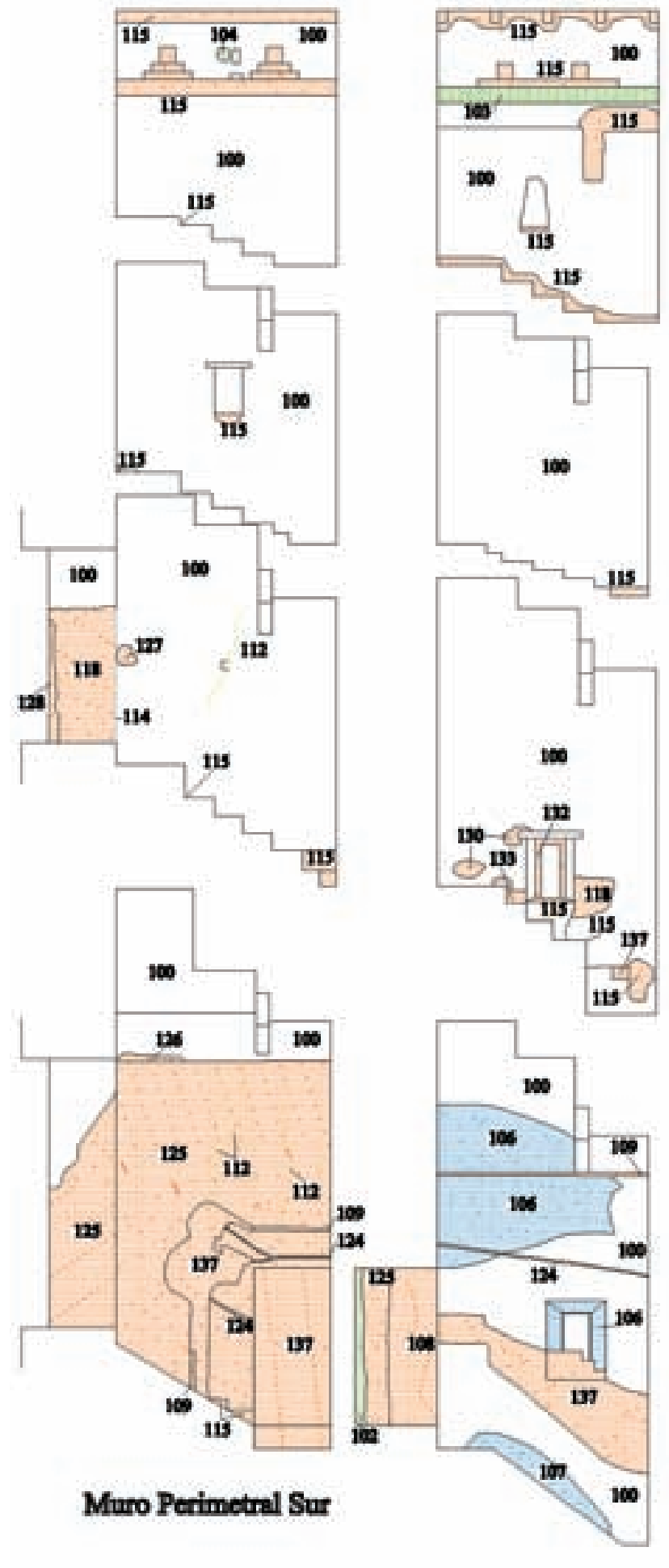

Muro Perimetral Oeste
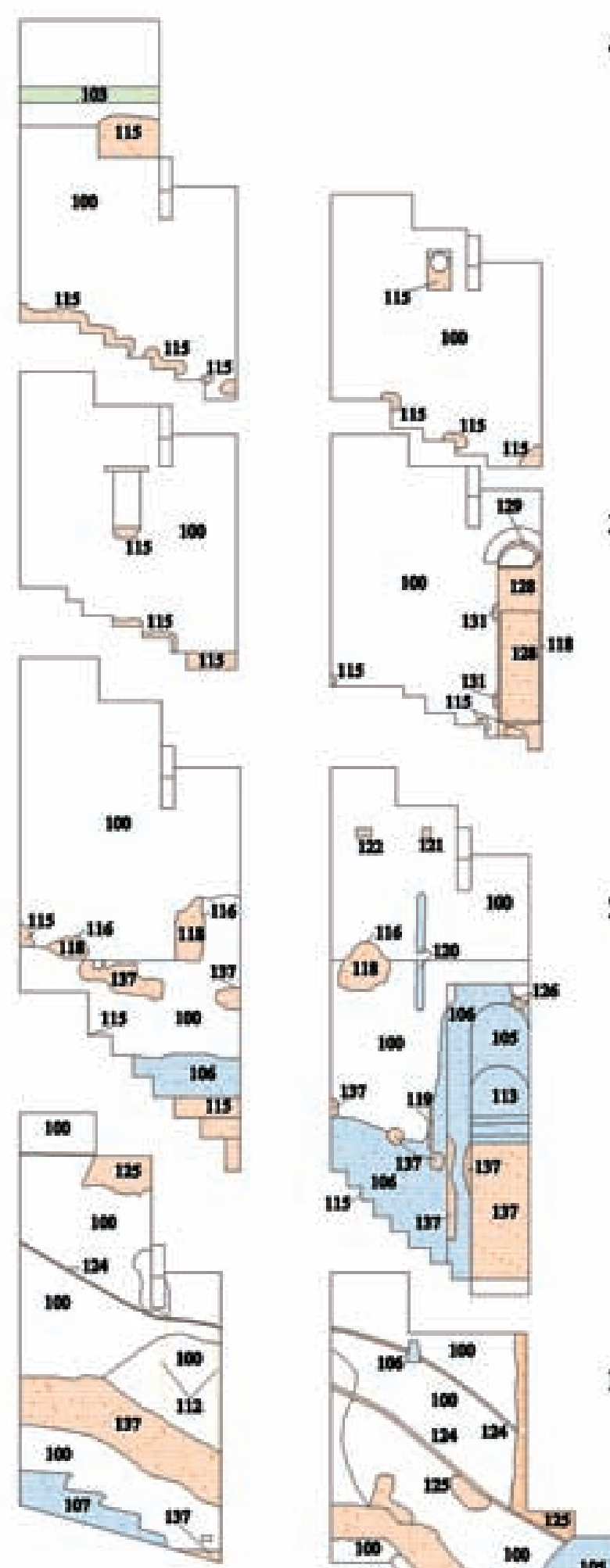

$3^{\circ}$

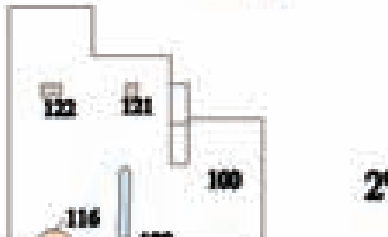

$2^{\circ}$

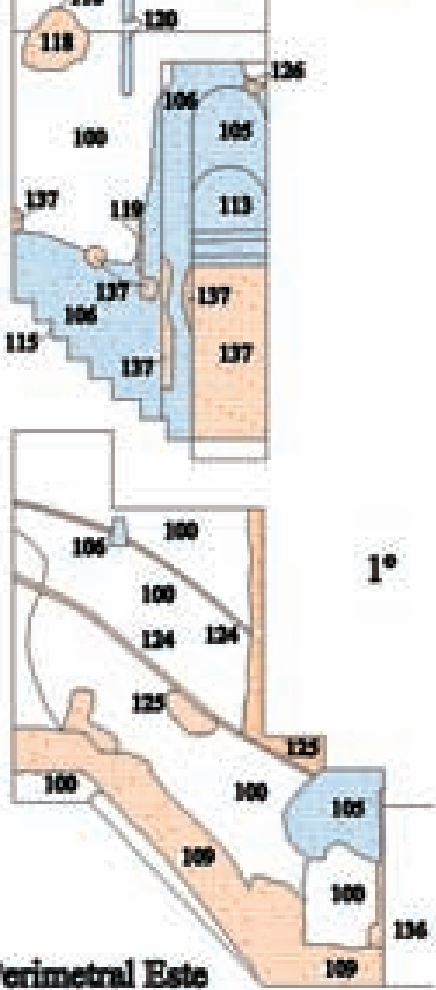

Muro Perimetral Bste

Muro Perimetral Norte

$0 \quad 1 \quad 2 \quad 3 \mathrm{~m}$

Figs. 19 y 20. Planos de lectura de paramentos 

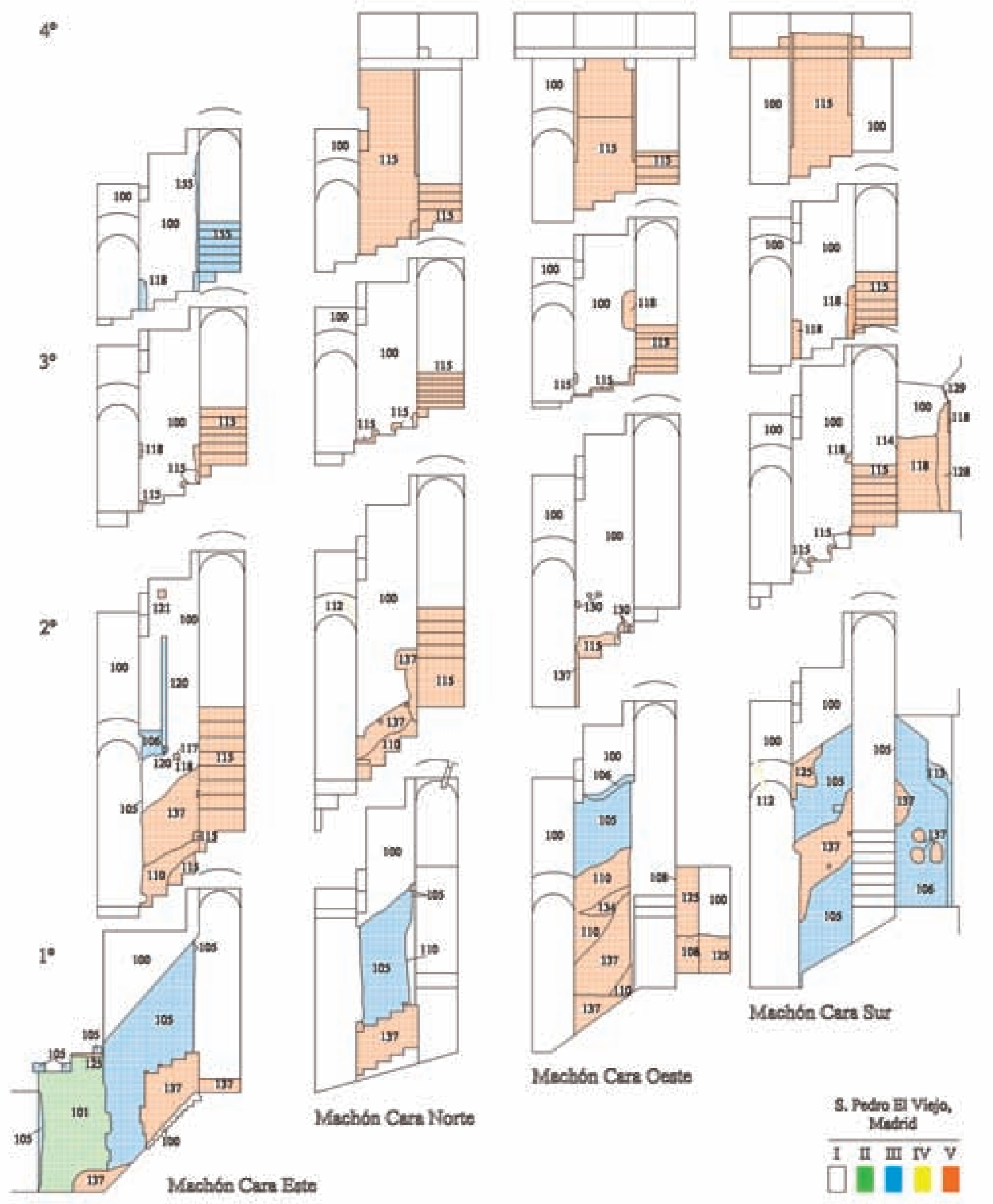

Mechon Cere Oeate

S. Podro Bi Viejo, Madrid I If III TV V ก 
La técnica constructiva de la torre indica el equilibrio buscado entre una construcción sólida y resistente y una construcción orgánica y flexible que pudiera hacer frente a posibles movimientos. A ello consideramos que responden sus características constructivas, como el remate de la arquería de la iglesia con el paño de su fachada norte y, especialmente, la mayor importancia dada a la obra de calicanto y la mayor pendiente de la escalera en sus primeras plantas, frente a la tendencia a la horizontalidad en las plantas superiores. Se utilizan las mesetas en la parte alta como elementos de trabazón del muro y el machón, mientras que, en la baja, la rigidez se la aporta el material de calicanto del muro perimetral.

\section{Bibliografía}

Abad Castro, M. ${ }^{a}$. C. 1991, Arquitectura Mudéjar Religiosa en el Arzobispado de Madrid, Caja Toledo, Toledo, 2 vols.

Ávila Jalvo, J. M. 1993, Estudio sobre la Torre de la Iglesia de San Pedro el Viejo (Consejería de Educación y Cultura. Comunidad Autónoma de Madrid), manuscrito.
Id. 1998, Tres tristes torres. Del Trovador de la Aljafería de Zaragoza, de San Pedro el Viejo de Madrid, de San Pedro ad Vincula de Vallecas, Cuadernos del Instituto Juan de Herrera de la Escuela de Arquitectura de Madrid 34.01, Cuadernos de Restauración 4.

FiTA, F. 1886, Madrid desde el año 1228, hasta el de 1234, Boletín de la Real Academia de la Historia, 8, 417 ss.

Gómez Moreno, M. 1927, La torre de S. Nicolás en Madrid, Archivo Español de Arte y Arqueología, 1, 129 ss.

GuARÁs, B. 2002, Estudio Petrológico de Morteros de la Torre Mudéjar de San Pedro el Viejo de Madrid, manuscrito.

ÍnigueZ AlmeCH, F. 1971, La iglesia parroquial de S. Salvador en Madrid, Boletín de la Real Academia de San Fernando, 32, 106 ss.

Montero Vallejo, M. 1987, El Madrid Medieval, Madrid.

Id. 1990, Madrid Musulmán, Cristiano y Bajo Medieval, Madrid.

Nuere, E. 1994, La armadura de lazo de la madrileña iglesia de San Pedro, en Boletín de la Real Academia de Bellas Artes de San Fernando, 176-194.

Pavón Maldonado, B. 1984/85, Arqueología y urbanismo medieval en Madrid. De la Almudayna árabe a la torre mudéjar de S. Nicolás, Awrāq Yâdida, 78, 231-278.

Quintana, J. DE. 1629, Historia de la Antigüedad, Nobleza y Grandeza de la Villa de Madrid (1954).

Retuerce Velasco, M. 1998, La Cerámica Andalusi de la Meseta, Zaragoza, 2 T.os.

Tormo y Monzo, E. 1927, Las Iglesias del Antiguo Madrid, Madrid (1979). Torres Balbás, L. 1949, Arte Almohade. Arte Nazarí. Arte Mudejar, "Ars Hispaniae», IV, Madrid. 


\section{APÉNDICE}

\section{San Pedro el Viejo (Madrid). Listado de actividades}

E. = Etapas. SE = sin etapa.

En la columna «A (UE)», las actividades (centenas) se marcan en negrita. Si se trata de una Actividad formada por una sola Unidad Estratigráfica (millares), su número se coloca a continuación entre paréntesis. Si la forman varias, se listan a continuación. Las columnas de "Anterior a» $\mathrm{y}$ "Posterior a», se refieren solo a Actividades. Se incluyen en ellas (subrayadas) referencias con A de la misma etapa (aunque en realidad son coetáneas) cuya relación de antero/posterioridad corresponde de hecho a sus UE. La columna de «Plano» se refiere a la memoria original y para su uso se puede seguir la siguiente equivalencia: Plano 1, 1. ${ }^{a}$ planta del muro perimetral; Plano 2, 2. ${ }^{a}$ planta del muro perimetral; Plano 3, 3. ${ }^{a}$ planta del muro perimetral; Plano 4, 4. ${ }^{a}$ planta del muro perimetral; Plano 5, 1. ${ }^{a}$ planta del machón central; Plano 6, 2. ${ }^{a}$ planta del machón central; Plano 7, 3..$^{a}$ planta del machón central; Plano 8, 4. ${ }^{a}$ planta del machón central; Plano 9, Exterior torre muro oeste; y Plano 10, Exterior parte baja muros norte y sur. Los planos 9 y 10 no se recogen en el presente trabajo.

\begin{tabular}{|c|c|c|c|c|}
\hline E & A (UE) & Anterior a & Posterior a & Plano \\
\hline 1 & $\begin{array}{l}100 \text { Construcción de la torre original } \\
1000 \text { Fábrica original } \\
1007 \text { Restos de ladrillo entre vigas, puerta de torre, planta } 1 .^{\mathrm{a}} \text {, muro } \mathrm{S} \\
1010 \text { Chapado de ladrillo, puerta de torre, planta } 1 .^{\mathrm{a}} \text {, jamba E } \\
1016 \text { Asiento del arco, planta } 1 .^{\mathrm{a}} \text {, muro } \mathrm{S} \\
1017 \text { Impronta de madera, planta } 1 .^{\mathrm{a}} \text {, muro N } \\
1022 \text { Huella de muro de ladrillo, planta } 1 .^{\mathrm{a}} \text {, muro N } \\
1023 \text { Fragmento de atoques, machón, planta } 1 .^{\mathrm{a}} \text {, lado E } \\
1035 \text { Huecos en jamba N puerta acceso coro, planta } 2 .^{\mathrm{a}} \text {, muro E } \\
1043 \text { Madera en machón, planta } 2 .^{\mathrm{a}} \text {, lado E } \\
1046 \text { Cabeza de viga entre fábrica calicanto y ladrillo, planta } 2 .^{\mathrm{a}} \text {, muro N } \\
1052 \text { Posible mechinal, planta } 2 .^{\mathrm{a}} \text {, muro } \mathrm{S} \\
1086 \text { Relleno de calicanto, planta } 1 .^{\mathrm{a}} \\
1087 \text { Estructura de ladrillo y barro, planta } 1 .^{\mathrm{a}} \\
1092 \text { Dos maderas sobre viga de cubierta } 103 \text {, planta } 4 .^{\mathrm{a}} \\
1094 \text { Iglesia, nave } \mathrm{N}, \text { muro N }\end{array}$ & $\begin{array}{l}\frac{100 / 17}{11} 19 / 27129 / 33 \\
136 / 8141 / 50153 \\
155 / 63165 / 71\end{array}$ & $\underline{100}$ & $1 / 10$ \\
\hline II & $\begin{array}{l}\mathbf{1 0 1} \text { Reforma jamba O, puerta de torre, planta } \mathbf{1}^{\mathbf{a}} \text {, esquina SE } \\
1002 \text { Superficie de enjarje entre fábrica original y forro que adosa } 1003 \\
1003 \text { Forro que se adosa a la fábrica original, junto a puerta de entrada } \\
\mathbf{1 0 2} \text { Fábrica de ladrillo, jamba O puerta cofradía, planta 1. a, muro } \mathbf{S}(1026) \\
\mathbf{1 0 3} \text { Reforma de cubierta (1059) } \\
\mathbf{1 0 4} \text { Agujero circular sobre viga, planta } \text { 4. }^{\text {a }} \text {, muro } \mathbf{S}(1060)\end{array}$ & $\begin{array}{l}\frac{101}{105} \\
108 \\
115\end{array}$ & $\begin{array}{l}100 \\
100 \\
100 \\
100\end{array}$ & $\begin{array}{l}1 \\
4 \\
4\end{array}$ \\
\hline III & 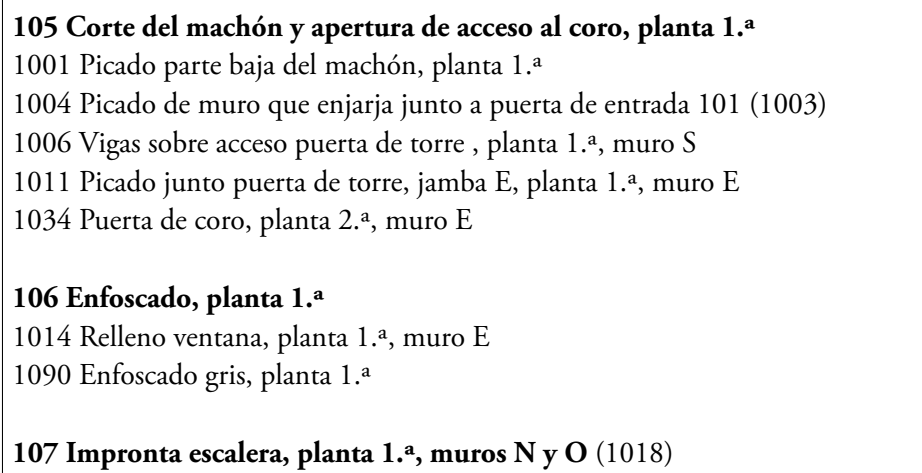 & $\begin{array}{l}\underline{106} 110 \underline{113} 125 / 6 \\
136 / 7 \\
\\
108 / 9112 / \underline{3} \underline{120} 125 \\
137 \\
\underline{137}\end{array}$ & $100 \underline{105} 124$ & 125 \\
\hline
\end{tabular}




\begin{tabular}{|c|c|c|c|c|}
\hline E & $\mathbf{A}$ (UE) & Anterior a & Posterior a & Plano \\
\hline & 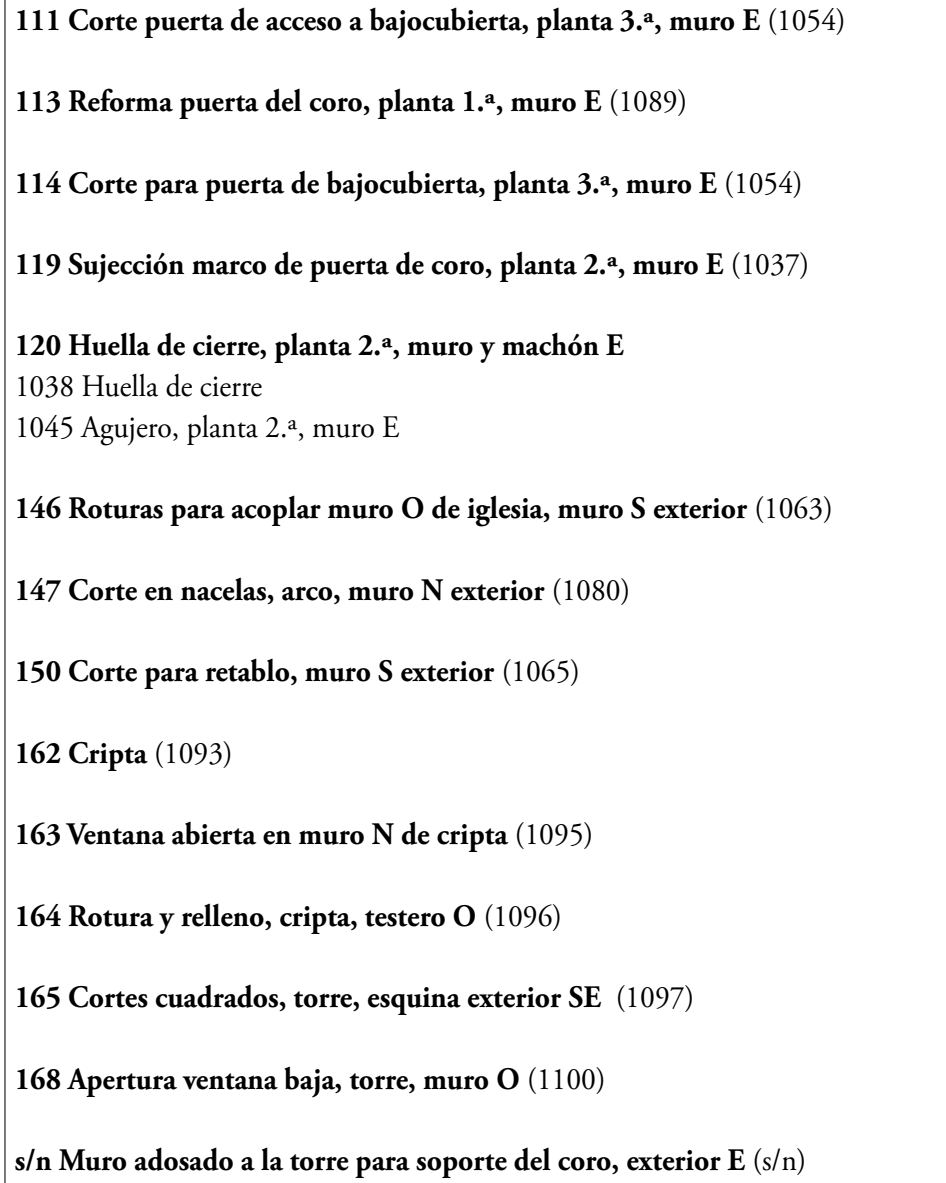 & $\begin{array}{l}151 \\
\underline{163164}\end{array}$ & $\begin{array}{l}100 \\
100 \\
100 \\
100 \\
100 \underline{162} \\
100 \\
100\end{array}$ & $\begin{array}{l}10 \\
10\end{array}$ \\
\hline IV & 112 Grietas de movimiento de la torre (1025) & 140144 & 100106155 & 125610 \\
\hline V & 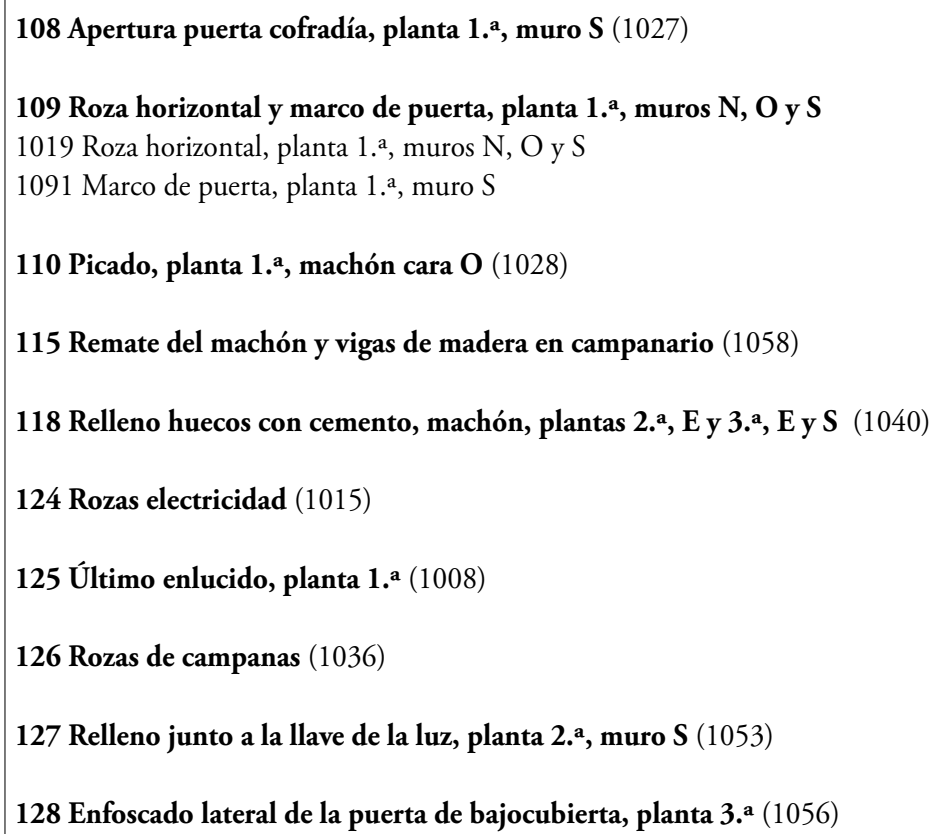 & $\begin{array}{l}\underline{134} \\
\underline{133} \underline{171} \\
\underline{128} \underline{129} \\
\underline{125} \underline{137} \\
112 \underline{137} \\
\underline{125}\end{array}$ & $\begin{array}{l}100105 \\
100103 \\
100111114116 / 7 \\
100 \\
106 \underline{108} \underline{124} \underline{126} \\
100105 \\
100 \\
\underline{118}\end{array}$ & $\begin{array}{l}6 \\
48 \\
26 / 8 \\
1 \\
15 \\
12 \\
2 \\
236\end{array}$ \\
\hline
\end{tabular}




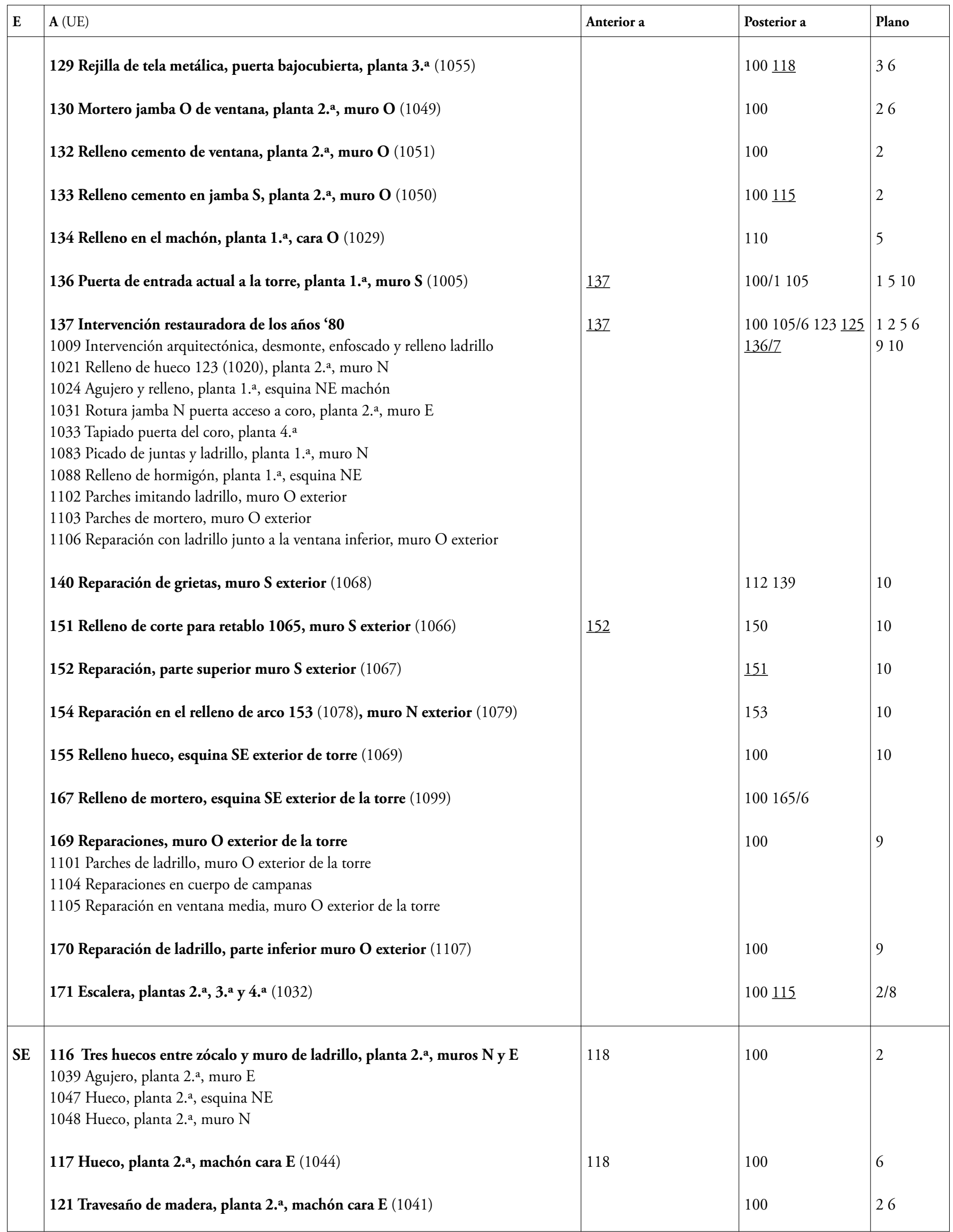




\begin{tabular}{|c|c|c|c|c|}
\hline E & A (UE) & Anterior a & Posterior a & Plano \\
\hline & 122 Hueco, planta $2 .^{\text {a }}$, muro de ladrillo E (1042) & & 100 & 2 \\
\hline & 123 Hueco a media altura, planta $2 .^{a}$, muro N (1020) & 137 & 100 & \\
\hline & 131 Hueco en ventana, jamba $N$, planta 3.a (1049) & & 100 & 3 \\
\hline & 138 Hueco, muro S exterior, parte inferior central (1070) & $\underline{139}$ & 100 & 10 \\
\hline & 139 Relleno de hueco 138, muro $S$ exterior, parte inferior central (1071) & 140 & 138 & 10 \\
\hline & 141 Hueco, muro $N$ exterior, esquina $\mathrm{O}(1085)$ & & 100 & 10 \\
\hline & 142 Roza horizontal, muro $S$ exterior $(1061)$ & & 100 & 10 \\
\hline & 143 Corte para puerta, jamba $\mathbf{O}$ del arco, nave $\mathbf{N}(1084)$ & & 100 & 10 \\
\hline & 144 Tres roturas, muro $S$ exterior, parte alta $(1062)$ & 112 & 100 & 10 \\
\hline & 145 Corte en jamba $\mathrm{E}$ del arco, muro $\mathrm{N}$ exterior (1081) & & 100 & \\
\hline & 148 Picado, muro S exterior (1064) & 152 & 100 & 10 \\
\hline & 149 Picado jambas de arcos, muro N exterior (1082) & & 100 & 10 \\
\hline & 153 Corte del arco, muro $\mathrm{N}$ exterior (1078) & 154 & 100 & 10 \\
\hline & 156 Corte, muro $S$ exterior $(1074)$ & & 100 & 10 \\
\hline & 157 Corte y relleno bajo relleno de retablo 151 , muro $S$ exterior (1072) & & 100 & 10 \\
\hline & 158 Hueco junto jamba E, parte superior, muro N exterior (1077) & & 100 & 10 \\
\hline & 159 Corte y relleno, parte baja, muro $S$ exterior $(1073)$ & & 100 & 10 \\
\hline & 160 Pareja de huecos, muro N exterior (1075) & & 100 & 10 \\
\hline & 161 Huecos, parte superior, muro $\mathbf{N}$ exterior (1076) & & 100 & 10 \\
\hline & 166 Corte diagonal, esquina SE exterior de la torre (1098) & 167 & 100 & \\
\hline
\end{tabular}

